Accepted refereed manuscript of:

Phillips W, Clark A \& Silverstein SM (2015) On the functions, mechanisms, and malfunctions of intracortical contextual

modulation, Neuroscience and Biobehavioral Reviews, 52, pp. 1-20.

DOI: $10.1016 / j$. neubiorev.2015.02.010

(C) 2015, Elsevier. Licensed under the Creative Commons Attribution-NonCommercialNoDerivatives 4.0 International http://creativecommons.org/licenses/by-nc-nd/4.0/ 


\author{
Pre-publication version of a paper published in: \\ Neuroscience and Biobehavioral Reviews 52 (2015) 1-20 \\ http://dx.doi.org/10.1016/j.neubiorev.2015.02.010
}

\title{
On the functions, mechanisms, and malfunctions of intracortical contextual modulation
}

\author{
W. A. Phillips ${ }^{1}$, A. Clark², and S. M. Silverstein ${ }^{3}$
}

\begin{abstract}
A broad neuron-centric conception of contextual modulation is reviewed and re-assessed in the light of recent neurobiological studies of amplification, suppression, and synchronization. Behavioural and computational studies of perceptual and higher cognitive functions that depend on these processes are outlined, and evidence that those functions and their neuronal mechanisms are impaired in schizophrenia is summarized. Finally, we compare and assess the long-term biological functions of contextual modulation at the level of computational theory as formalized by the theories of coherent infomax and free energy reduction. We conclude that those theories, together with the many empirical findings reviewed, show how contextual modulation at the neuronal level enables the cortex to flexibly adapt the use of its knowledge to current circumstances by amplifying and grouping relevant activities and by suppressing irrelevant activities.
\end{abstract}

Keywords: cognitive coordination; contextual modulation; amplification; suppression; Gestalt grouping, predictive processing

1. Department of Psychology, University of Stirling, FK9 4LA, Scotland, UK

2. School of Philosophy, Psychology, and Language Sciences, University of Edinburgh, EH12 5AY, Scotland, UK

3. Rutgers Biomedical and Health Sciences, Piscataway, New Jersey, USA

Correspondence:

Steven M. Silverstein

Rutgers Biomedical and Health Sciences, Piscataway, New Jersey, USA

steven.silverstein@rutgers.edu, tel: 1.732-235-5149

Running title: Contextual modulation 


\section{Introduction}

All life requires the coordination of many specialized activities so that their joint activities sustain the existence of the organism as a whole. The necessity of both specialization and coordination is particularly clear in the cerebral neocortex. A central achievement of the cognitive and neurosciences has been to map the extensive functional specializations that exist within as well as between cortical regions. All those specialized activities must be adequately coordinated, however, if they are to produce coherent percepts, thoughts, and actions that are well-adapted to current circumstances and long-term goals. How this is achieved has been, and still is, a major issue within the cognitive and neurosciences. The dynamic coordination required involves several different levels of organization. They range from the level of pyramidal cells and local cortical microcircuits up to the level of macroscopic subsystems, such as the executive functions of pre-frontal cortex. The focus of this review is on that lower level of organization, which we will argue is relevant to all cortical regions.

The central hypothesis reviewed here is that much of the coordination required is achieved by widely distributed processes of contextual modulation. They amplify the transmission of signals that are relevant to current circumstances, and suppress those that are irrelevant. They also synchronize selected signals into coherent sub-sets. The notion of 'contextual modulation' can be defined so as to refer only to a narrowly specified set of phenomena, such as surround suppression or figure-ground segregation in vision, for example. We define it in a broader way, however. In that broader form it refers to neuronal processes and mechanisms that underlie a wide range of phenomena such as predictive processing, Gestalt grouping, contextual disambiguation, selective attention, and cognitive control. Section 2 is devoted to presenting that broader conception in detail. Evidence for it from a wide variety of methodologies is then reviewed and assessed in the following sections.

Figure 1 provides a simplified illustration of how contextual modulation operates in broad terms. It shows the driving feedforward inputs as coming from a narrowly specified set of sources. In primary sensory regions they come predominantly from the specific thalamic nucleus that relays the sensory information to which each microcircuit is selectively sensitive. In higher regions they come from a narrowly specified sub-set of microcircuits within other cortical regions. The modulatory contextual inputs to the local microcircuits come from a wide diversity of sources, such as distant locations within the same region, feedback from higher regions, non-specific thalamus, and various other sources. Evidence reviewed below suggests that in some psychopathological conditions, such as schizophrenia, contextual modulation is less effective, which leads to patterns of neural activity that are less coherent overall and behavior that is less well adapted to current circumstances and more disorganised or impulsive. 


\section{Level C}

\section{Level B}

\section{Level A}

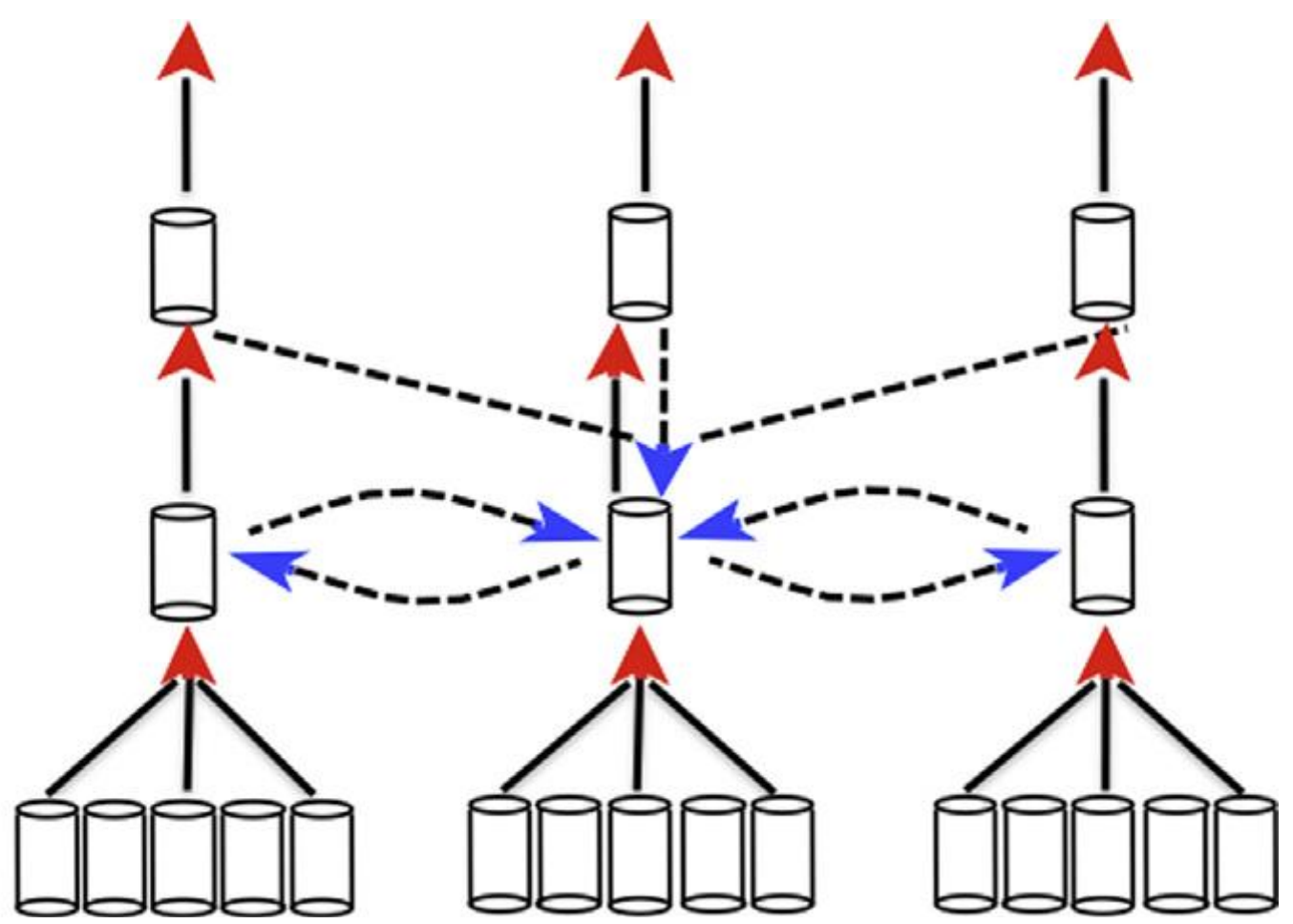

Fig. 1. Contextual interactions in a hierarchy of cortical regions composed of many local microcircuits. A few of the many microcircuits are shown here as small cylinders. Feedforward pathways are depicted by solid lines, and contextual interactions by dotted lines, which are shown here only for the central microcircuit at Level B, though all have similar connectivity. The contextual interactions include both feedback from higher regions and recurrent interactions with other microcircuits at the same level. The recurrent interactions include those between microcircuits that are in the same cortical region but which have non-overlapping feedforward inputs. They also include interactions between different regions that are at equivalent levels of the hierarchy. The contextual interactions modulate response to the feedforward drive so as to produce patterns of activity that are coherent across the network as a whole. The internal anatomy of the microcircuits and the convergenceand divergence of feedforward pathways are not shown.

The minimal computational requirements for cortical microcircuits that use contextual inputs to modulate response are shown in Figure 2. The modulatory inputs must be integrated separately before being used to modulate response to the integrated driving inputs (Kay and Phillips, 2011).The two separate sites of integration shown in Figure 2A could be implemented by two distinct sets of neurons. Section 3 will cite evidence that local cortical circuits contain neurons that integrate inputs from various contextual sources before using their outputs to either amplify or suppress the responses of other cells in the local circuit. There is also evidence for modulatory interactions within pyramidal cells, however, and this is shown in Figure 2B. That evidence will also be reviewed in Section 3. Until recently it has been widely assumed in the cognitive and neurosciences that, from a functional point of view, neurons can be adequately conceived of as simply adding up all of their excitatory and inhibitory inputs and transmitting an axonal spike if that integrated value exceeds a threshold. Though networks built from such 'integrate and fire' neurons can do a lot, there is now evidence that pyramidal cells have two distinct sites of integration with input to one site modulating response to the driving input at the other site. We will refer to such processes as 'apical amplification' because they amplify response to the basal and perisomatic inputs by transmitting signals down the apical trunk of the pyramidal cell. 

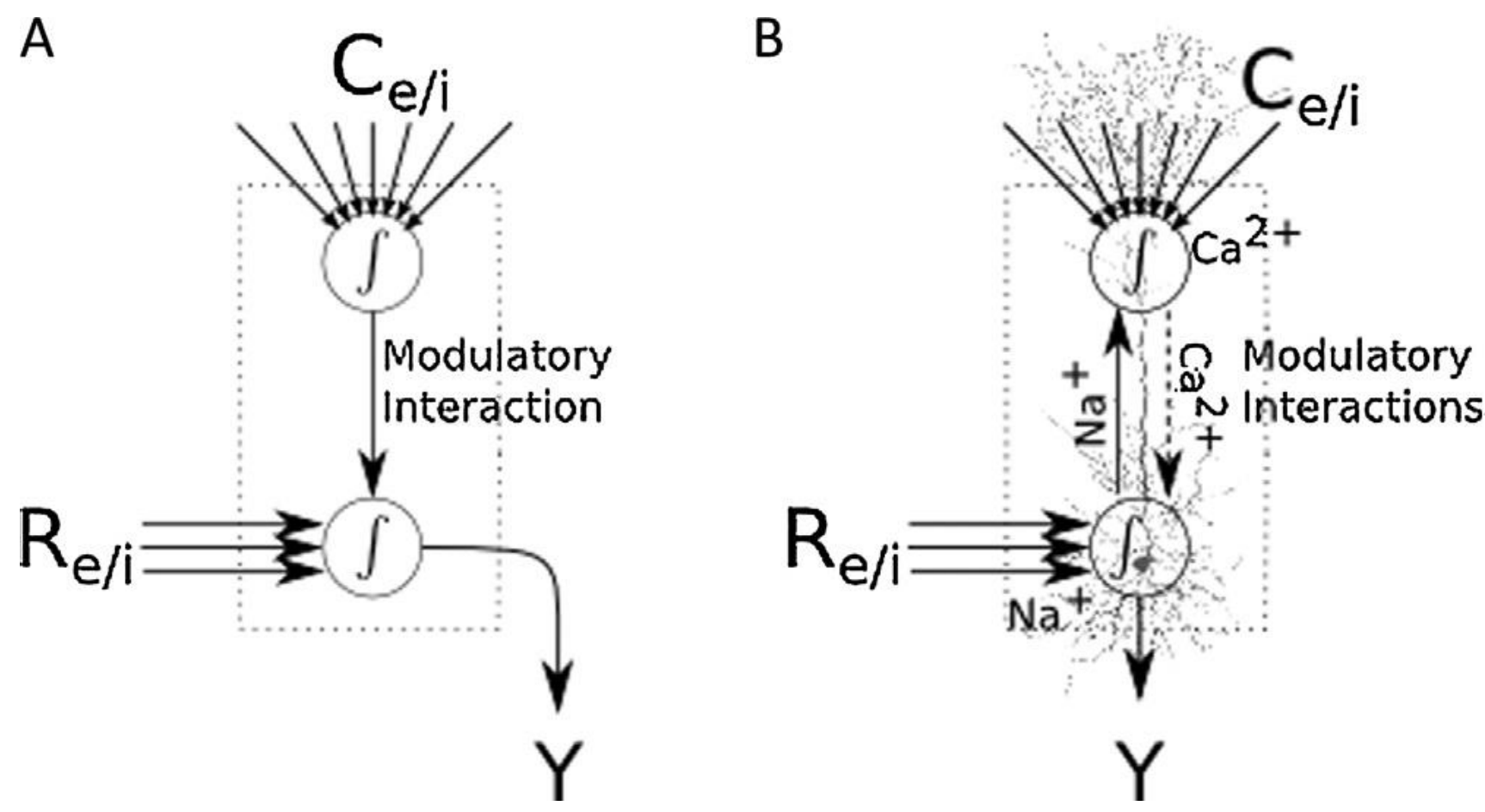

Fig. 2. Minimal computational requirements of local cortical microcircuits that use contextual inputs (C) to modulate response to their driving receptive field inputs( $R)$, and one possible mechanism that could meet these requirements within pyramidal cells. (A) The inputs labelled $\mathrm{R}$ and $\mathrm{C}$ are both composed of excitatory and inhibitory inputs (e/i). Excitatory inputs arise from local and long-range connections. Inhibitory inputs arise from various inhibitory interneurons in the local microcircuit. The inputs $\mathrm{R}$ are assumed to arise from narrowly defined sources, and the inputs $\mathrm{C}$ from a wide variety of sources. Circles indicate sites of integration. (B) As part A except that here the modulatory interactions are shown as operating within a single Layer 5 pyramidal cell. The two sites of integration are shown overlaid on a computer-generated sketch of a pyramidal cell with two sites of integration, one at the soma and one at the top of the apical trunk. The driving inputs arrive via basal and perisomatic synapses. The modulatory inputs arrive via synapses on the tuft dendrites at the top of the apical trunk. Na+indicates the somatic integration site that initiates sodium spikes which backpropagate up the apical trunk in addition to forming axonal action potentials. $\mathrm{Ca}^{2+}$ indicates the apical integration site initiating calcium spikes that amplify response. Source: Layer 5 cell created with the TREES toolbox (Cuntz et al., 2010), courtesy of Hermann Cuntz.

Our review relates contextual modulation to psychoses such as schizophrenia because the disorganization, incoherence, and reduced context-sensitivity in such disorders provide a window on the role of contextual modulation in mental life (Phillips and Silverstein, 2013). The many studies of synaptic malfunction in schizophrenia also cast light on the mechanisms that are impaired. It is wellknown that the aetiology and symptomatology of schizophrenia spectrum disorders have such great heterogeneity that they are very difficult to comprehend. Such disorders may be comprehensible at the level of a common underlying pathophysiology, however (Mitchell et al., 2013). We will therefore re-assess the hypothesis that impairment of contextual modulation is such a pathophysiology. For an introduction to and commentary on an earlier version of that hypothesis see Phillips and Silverstein (2003).

If contextual modulation is of relevance to many different cognitive domains then it should be possible to formally specify its capabilities and long-term goals in a domain-free conceptual framework (Wibral, et al., in preparation). That is done by the theory of coherent infomax, which proposes a general account of cortical function in which contextual modulation plays a central role that is crucial to both learning and processing (Phillips, Kay and Smyth, 1995; Kay, Floreano and Phillips, 1998; Kay and Phillips, 2011). Contextual modulation as conceived within that theory is 
therefore examined further here. For an introduction to, and commentary on, an early version of that theory see Phillips and Singer (1997).

Here we re-assess that conception of contextual modulation in the light of recent empirical and theoretical advances. Three groups of empirical findings are of particular importance. The first provides evidence for intracellular mechanisms by which contextual inputs can modulate response to driving inputs. In one such mechanism contextual inputs to the apical tuft of pyramidal cells amplify output of the whole cell given sufficient drive from the basal and perisomatic synapses, as shown in Figure 2B. It has been argued that this mechanism, referred to as backpropagation activated calcium spike firing (BAC firing), plays a major role in cortical function (Larkum, 2013; Major, Larkum and Schiller, 2013). Evidence for that claim is reviewed in Section 3. The second group of findings provides further evidence on the role of synchronized spiking activities, particularly at gamma, beta, and theta frequencies (e.g. Fries, Nikolic and Singer, 2007; Vinck, Womelsdorf and Fries, 2013, Bastos et al 2015). The third includes findings that have been made possible by the optogenetic control of neuronal activity with high levels of temporal and spatial precision (e.g. Atallah, 2012; Pouille et al., 2009). The most relevant theoretical advance is that based on free energy reduction and hierarchical predictive processing. For an introductory outline see Friston (2009). For a more advanced summary see Friston (2010). For a broad overview of its psychological and philosophical significance see Clark (2013a, b) and the associated commentaries.

The body of this paper is organized as follows. Section 2 says what we mean by 'contextual modulation'. It is necessary to do this because, though the phrase is often used in the cognitive and neurosciences, it does not yet have a single commonly accepted definition. Section 3 reviews neurobiological evidence on contextual modulation and its intracellular and local-circuit mechanisms. It outlines evidence for three basic forms of contextual modulation, i.e. amplification, suppression, and dynamic grouping by synchronization. Section 4 reviews behavioural and computational studies of basic cognitive functions that depend upon these various forms of contextual modulation. Section 5 summarizes evidence that those cognitive functions and their putative neuronal mechanisms are impaired in schizophrenia. Section 6 discusses the long-term biological objectives of contextual modulation at the level that Marr (1982) called 'computational theory'. Formally expressed objectives as seen from each of three information-theoretic perspectives are considered. The first is that of free energy theory, i.e. that of a whole neural system operating within an ecological niche. The second is that of coherent information theory, i.e. that of pyramidal cells, and their associated local circuit neurons, operating within a neural system. The third is that of a person's directly lived experience. We argue that the formal objectives hypothesized by each of those three perspectives, though different, are fundamentally compatible. Finally, Section 7 briefly considers difficulties, limitations, and prospects for this broad neuron-centric conception of contextual modulation.

\section{A neuron-centric conception of contextual modulation}

The aim of this section is simply to make clear what we mean by 'contextual modulation' at the neuronal level. If we did not do so then the claim that it is central to cortical computation would have little substance. Surprisingly, we cannot do this by referring to a widely accepted and rigorous definition because we know of none that is adequate.

Though much used in the cognitive and neurosciences, the terms 'modulation' and 'context' do not have commonly agreed meanings. The term 'modulation' usually refers to some change in the function that relates neuronal output to the strength of its driving input. Those changes can take various forms, however, depending on whether they relate to the threshold, slope, or asymptote of the function. 'Modulation' is sometimes used to refer specifically to changes in slope, but it is also sometimes used more broadly. The term 'context' is even more ambiguous (Park, et al., 2003). Sometimes it is used to refer to information coming from a particular source in the system architecture, e.g. from regions that are higher in the hierarchy. It is also often used to refer 
specifically to concurrent stimulus information arising from a particular source, such as that from surrounds of the distal classical RF, or from other modalities. In yet other cases it is used to refer to information with particular semantic content, such as information about the place at which reinforcing events occur. It is also sometimes used to refer specifically to information that is held in working memory. Finally, the notion of 'context' is often used in an intuitive sense to refer to information about things other than whatever the experimenter or the subject take to be the 'target' or focus of current activities. It is therefore necessary for us to provide a more rigorous conception of contextual modulation. We call it 'neuron-centric' because, in the spirit of Fiorillo (2012), it is defined strictly from the point of view of a local microcircuit or pyramidal cell, i.e. in terms of the distinct kind of effect that it has on the output of that cell or microcircuit, assuming the use only of information available to it, as shown in Figures 1 and 2.

This conception is founded on a distinction between the effects of two classes of input, i.e. primary driving inputs that specify classical receptive field (RF) selectivity, and contextual inputs that modulate response to the RF input. In the case of cells in visual cortex, for example, input arising from the distal classical RF provides the primary drive, and contextual field (CF) input arising from various other sources modulates response to the primary drive. Our distinction between RFs and CFs has much in common with that between drivers and modulators proposed by others (e.g. Lee and Sherman, 2010), and it has often been argued previously that contextual modulation has a central role in cortical function (e.g. Salinas and Their, 2000; Salinas and Sejnowski, 2001; Lamme, 2004; Haider and McCormick, 2009; Gilbert and Sigman, 2007; Major, Larkum and Schiller, 2013). Though our conception of contextual modulation differs in some ways from those other conceptions, our search here is for conclusions on which we all converge.

Notions of contextual modulation at the cellular level often imply that one variable, the context, affects transmission of information about another. For example, Salinas and Sejnowski (2001) argue that gain modulation is the basis for a general class of computations that is revealed when one input, the modulatory one, affects the gain or sensitivity of a pyramidal cell to its RF input, without fundamentally modifying its RF selectivity. RF input can generate output action potentials by itself when sufficiently strong, whereas modulatory inputs cannot usually generate action potentials by themselves, but can have large effects given some RF input (e.g. Lund, Angelucci and Bressloff, 2003). Therefore, we define contextual inputs as those that change the cell's response to its RF input but which do not by themselves usually generate action potentials. Thus, this conception defines contextual inputs by their modulatory effects not by their semantic content, locus of origin within the network architecture, or relations to current or previous sensory input. The following sub-sections further clarify this view.

\subsection{Contextual modulation is widespread throughout many regions of neocortex}

Evidence reviewed below suggests that the distinction between modulatory and driving inputs applies to many different cortical regions. The distinction is supported by intracellular studies showing that pyramidal cells with clearly distinct receptive field selectivities can be directly connected while still maintaining their own distinctive selectivity (Schummers, Marino and Sur, 2002; Monier et al., 2003). A similar distinction has been made by several others (e.g Salina and Sejnowski, 2001; Lee and Sherman, 2010; Larkum, 2013). We assume that axonal action potentials act as drivers at some of their projective sites, and as contextual modulators at others.

Though much of the electrophysiological evidence for contextual modulation comes from sensory or perceptual regions, not all of it does, and there are good grounds for assuming it to be much more widespread. Context-sensitivity is required for many cognitive tasks so it is likely to be present in many cortical regions. Furthermore, the assumption that computational capabilities such as contextual modulation have a general role in cortical computation is supported by evidence for a 'canonical cortical circuit' that is common to many, though not necessarily all, regions of neocortex (Douglas and Martin, 2007, 2008). Though the anatomical evidence on microcircuitry within cortical 
columns is still far from complete, there is evidence for cortical columns that are physiologically discrete in the sense that they code for distinct local regions of multidimensional feature space (Lund, Angelucci and Bressloff, 2003). The point of central relevance here is that contextual modulation at the cellular and microcircuit level is common to many different cortical regions. Much further evidence for this is reviewed in detail below.

\subsection{Modulatory inputs are defined by the effects that they have, not by where they come from} It is often assumed that contextual modulation can be identified with feedback or top-down interactions, but this can be misleading as made clear by Markov and Kennedy (2013). Though many long-range lateral and feedback connections may be modulatory, some may be driving (e.g. Fiorani, et al., 1992; Sugita, 1999; Fitzpatrick, 1996; Muckli and Petro, 2013). There is also in vitro evidence that, in the cortex of mice, V1 and V2 have both driving and modulatory connections in both directions (De Pasquale and Sherman, 2011), as do auditory regions A1 and A2 (Covic and Sherman, 2011). Thus, those studies show that driving and modulatory synapses can be distinguished independently of their position in the network architecture.

Driving inputs cannot in general be identified with those arising from current sensory input because that would imply that inputs to pyramidal cells arising from memory or other internal processes cannot be driving. They can be, and the use of context to make those activities coherent is just as important as it is when the activity is externally driven (e.g. Martin and Pacherie, 2013).

\subsection{Contextual modulation can take several forms}

Contextual modulation can change the threshold, slope, or asymptote of the function relating neuronal output to the driving input in various ways. This includes changes to either input gain or response gain, but requires output to be at or near zero when there is no driving input. We do not identify it with non-linear interactions in general because it is possible that some RF selectivity depends on other forms of non-linear interaction (Jadi et al., 2014), such as the multiplicative interactions required to compute coordinate transformations (Phillips and Silverstein, 2013).

\subsection{Contextual modulation can alter the precision or sharpness of tuning}

On both theoretical and empirical grounds, it can be assumed that, although contextual modulation does not alter the distal variable that is estimated by the local processor, it can alter the precision or coarseness of that estimate, as shown by changes in the width of the tuning function without shifts in the location of its peak.

\subsection{Contextual modulation can have large sub-threshold effects without generating axonal action potentials}

There is evidence that contextual inputs can by themselves produce large sub-threshold effects on distal dendritic currents without generating action potentials. Thus, in the absence of RF input, they could have substantial effects on dendritic currents, local field potentials (LFPs), or macroscopic measures even if they are not sufficient to generate axonal action potentials. Paradigms that use macroscopic neuroimaging to investigate the effects of feedback by eliminating sensory feedforward information, e.g. as reviewed by Muckli and Petro (2013), therefore need to distinguish effects on output action potentials from effects on distal dendritic currents.

\subsection{The selective sensitivity specified by driving inputs is labile}

In agreement with Salinas and Sejnowski (2001), we define modulation as affecting sensitivity without fundamentally modifying RF selectivity. We know that selectivity is not rigidly fixed, however. This lability even includes the ability to convert modulatory synapses to driving synapses if the original RF input is removed for long enough (e.g. Das and Gilbert, 1995). To be useful, however, such change requires appropriate modifications at the sites to which the cell projects. That 
requirement may not be easily met, however, so our working assumption is that, at any given time, synapses function either to specify RF selectivity or to modulate the cell's response to its RF input.

\subsection{Intracortical modulatory interactions must be distinguished from the effects of the classical neuromodulators}

The focus of this paper is specifically upon modulatory interactions within the cortex, rather than on modulation of cortical activities by sub-cortical systems, which has been reviewed in-depth by Dayan (2012). Intracortical modulation is emphasized here because many of the detailed cognitive contents that need to be coordinated via modulatory interactions are conveyed only by the activities of cortical neurons. Nevertheless, modulation of cortical activity by the classical sub-cortical neuromodulators shares with intracortical modulation the essential property of regulating signal transmission without corrupting the information transmitted by those signals.

\section{Contextual modulation of neuronal activity in the cortex}

The aim of this section is to review neurobiological studies of contextual modulation and its mechanisms. It is well-established that modulation and context-sensitivity are widely distributed across the cortex. Evidence for this ranges from electrophysiological studies of single cells to studies of population activity (see reviews by Schwartz, Hsu, and Dayan, 2007; Gilbert and Sigman, 2007; Lamme, 2004; Phillips and Silverstein, 2013; Silver, 2010). We distinguish three basic forms of contextual modulation and summarize evidence on the local circuit and intracellular mechanisms by which they are implemented. Though distinct, these three forms of modulation are variations on a common theme: amplification of relevant and suppression of irrelevant activities given the current context. The central problem throughout is that of specifying relevance; each form of contextual modulation makes a special contribution to solution of that problem.

\subsection{Modulation that amplifies responses to RF input}

Modulation that amplifies neuronal responses in primary visual cortex has been much studied in the case of response to short line elements that are displayed in the context of collinear flanking elements. Kapadia et al. (1995) found that, in awake macaque monkeys, the responses of complex cells in superficial layers of V1 to their preferred RF stimulus was often greatly facilitated by collinear flankers. They showed in detail how these effects depend upon the location and orientation of the flanking context relative to the central stimulus. They also showed that these effects were not due to the flankers encroaching within the distal RF of the recorded cell, but were due to modulatory interactions between cells with non-overlapping distal RFs. Such facilitation is greatest at target contrasts just above the cell's firing threshold (Polat and Norcia, 1996; Polat et al 1998), and includes cases where the flankers are placed at separations of 12 degrees or more (Mizobe et al, 2001), which is beyond the furthest extent of the cell's distal RF (Angelucci et al, 2002). This facilitation is mediated both by long-range intra-regional connections (Stettler et al, 2002) and by feedback from higher regions (Angelucci et al, 2002).

A recent study of the effects of collinear flankers recorded responses in cat V1 to stimuli designed to reveal the different contributions of feedforward, feedback, and horizontal (long-range intraregional) inputs to the cell (Kim and Freeman, 2014). Both facilitation and suppression were observed. Facilitation was predominant when the oriented grating at the RF centre was flanked by a small collinear grating placed beyond the classical RF. Suppression was predominant when the central stimulus was completely surrounded by an annulus. Both effects decreased with distance of the contextual stimuli from the stimulus at the centre of the cell's RF. Collinear intra-regional facilitation occurred slightly later than that produced by feedback, as predicted on the grounds that horizontal axons within V1 are thin and unmyelinated whereas feedback connections are myelinated and thicker. They also found that facilitation could occur with collinear flanking patches that were of 
lower contrast, size, or proximity than that required for suppression, which implies that the facilitation was not due to reduced suppression.

Amplifying modulation may also play a role in disambiguating visual motion signals. The temporal dynamics of a neural solution to the aperture problem in cortical motion-sensitive area MT of macaque cortex (Pack and Born, 2001; Pack et al. 2003) was found to agree with that implied by a computational strategy that amplifies coherent signals (Bayerl and Neumann, 2004; Perrinet and Masson, 2012). Note, however, that, as our conception of contextual modulation implies, the RF selectivity of motion detecting cells can be modelled without any use of modulatory inputs (Rust et al., 2006).

The role of amplification in object perception has been studied in many ways, including presentation of displays that can be seen either as a single coherent object or as a set of separate fragments. For example, an EEG study estimated the neural generator associated with seeing a stimulus as a single coherent object to lie in the lateral occipital cortex, a region long associated with object perception (Flevaris, Martínez, and Hillyard, 2013). When a probe stimulus was presented within the object boundary the P2d component of the ERP was amplified. This occurred with a latency of 184-212 ms, which is in accord with other evidence that amplification of responses to elements within a figure can occur without attention (Marcus \& Van Essen, 2002), though they can also be further enhanced by attention (Qui, Sugihara, \& von der Heydt, 2007).

Attention affects activity in many cortical areas by enhancing relevant activity, by sharpening RF selectivity, and by guiding perceptual and motor learning (Gilbert and Sigman, 2007). The amplifying effects of attention are similar to the disambiguating effects of stimulus contexts and to the effects of reducing ambiguity by increasing stimulus contrast (Treue and Martinez Trujillo, 1999; Williford and Maunsell, 2006). In some conditions the amplifying effects of attention occur with latencies of more than $100 \mathrm{msec}$ (e.g. Li, Piech and Gilbert, 2006; Roelfsema, Lamme, and Spekreijse, 1998; Bisley and Goldberg, 2006), but when attention can be oriented appropriately prior to stimulus onset its effects can be observed in LFPs prior to stimulus onset (Ramalingam et al., 2013). They can then affect the very first spike (Gilbert and Sigman, 2007). Similar effects of attention are found in auditory cortex, where RF selectivity remains remarkably stable, despite substantial attentional modulation (Massoudi, et al., 2013). This supports our assumption that attention is usually unable to generate feedforward activity by itself, as required to avoid selffulfilling prophecy. In addition to amplifying responses, attention can also facilitate contextual effects within V1 (e.g. Ito and Gilbert, 1999), particularly when stimuli are ambiguous (Ramalingam et al., 2013).

Amplification also occurs in higher cortical regions. Neuroimaging studies of people performing the Stroop task indicate that cognitive control mechanisms in pre-frontal cortex enhance performance by transiently amplifying cortical responses to relevant information in perceptual areas (Egner and Hirsch, 2005). Though there is still debate over how best to interpret cortical signals recorded during Stroop tasks, such findings suggest that attentional amplification of relevant features may be involved in the cognitive control exerted by prefrontal cortex.

By what mechanisms is such amplification achieved? In an earlier review of contextual modulation Lamme (2004) concludes that "contextual modulation is mediated by mechanisms that are far removed from those that shape and tune the local RF" (page 724). The most direct mechanism by which one pyramidal cell could amplify another's output is via direct connections between them. That would be both fast and energy efficient, and could have high local specificity. Such connections would have to be modulatory, however, and it has long been argued that this may be achieved via $N$-methyl-D-aspartate receptors (NMDARs) because their voltage-dependence makes them modulatory rather than driving (e.g. Phillips and Singer, 1997). They are highly expressed on pyramidal cells, and are closely involved in psychotic pathologies characterized by impairments of contextual modulation (Moghaddam and Javitt, 2012; Phillips and Silverstein, 2003; 2013). Furthermore, contextual modulation requires the transmission of a great deal of information, 
and about $75 \%$ of all cortical connections are directly between pyramidal cells (Braitenberg and Schuz, 1991). Direct evidence of a role for NMDARs in contextual modulation was shown by Self et al. (2012) using the well-established phenomenon of figure-ground modulation. This was almost abolished when NMDARs were blocked in V1, whereas the purely feedforward component of pyramidal cell response was largely unaffected. Therefore, NMDAR-mediated interactions between pyramidal cells may be involved in the direct monosynaptic amplification of coherently related activities.

There is also evidence of intracellular mechanisms for amplification, as indicated by Figure 2B. Evidence for an intracellular, NMDAR-dependent, mechanism that amplifies neuronal output was reported several years ago (e.g. Larkum, Zhu, and Sakmann, 1999). The far-reaching implications of such mechanisms are only now becoming clear, however (Larkum et al, 2009; Larkum, 2013; Major, Larkum and Schiller, 2013; Jadi et al, 2014). In vitro studies of rat somatosensory cortex show that, in addition to the somatic sodium spike initiation zone that triggers axonal action potentials, Layer 5 pyramidal cells have an initiation zone at the base of the apical tuft. If it receives adequate depolarization from the tuft dendrites and a backpropagated spike from the sodium spike initiation zone it triggers calcium spikes that travel down the apical trunk to the soma (Larkum, Zhu, and Sakmann, 1999). This backpropagation-activated calcium spike firing (BAC firing), has an explosive effect on the cell's output because it turns a single axonal spike into a brief burst of 2-4 spikes (Larkum, Senn, and Lüscher, 2004; Boudewijns et al, 2013). Tuft dendrites are well-positioned to implement contextual modulation because they receive their inputs from a wide variety of sources, including non-specific thalamic, long-range descending, and lateral pathways (Larkum, 2013). As it is remote from the soma, however, tuft input to Layer 5 cells has little effect on their output unless active dendritic currents, such as those triggered by BAC firing, occur. A consequence of this is that, on its own, input to the tuft has little effect on axonal action potentials. Inputs to the basal tree can produce axonal action potentials by themselves, however, and this response will be greatly amplified if they coincide with contextual input to the tuft. Calcium spikes are less likely if NMDAR channels are blocked, however, so any such contextual amplification would then be reduced or eliminated (e.g. Larkum et al, 2009; Larkum, 2013). Thus, evidence for this mechanism is in harmony with the evidence reviewed above for a major role of NMDARs in contextual modulation.

Though much of the research on BAC firing has been on layer 5 cells, there is evidence that apical amplification also occurs, in various forms, in other kinds of pyramidal cell, such as those in Layer 2/3 of rat somatosensory (Waters et al., 2003) and prefrontal cortex (Boudewijns, 2013). It is therefore important to note that although BAC firing may be a mechanism by which apical amplification is achieved in Layer 5 cells, it may be achieved in other ways in other classes of pyramidal cell (Palmer, et al., 2014). The crucial feature of these discoveries is that they show that pyramidal cells have intracellular mechanisms by which contextual amplification can be implemented, thus providing further evidence that it is widely distributed throughout the cortex.

Though their implications may be of great importance, dogma concerning such intracellular mechanisms is premature because researchers have barely begun to explore the vast space of possibilities (Major, Larkum and Schiller, 2013). For example, in addition to apical amplification of the whole cell's output, intra-dendritic contextual amplification may also occur. Behabadi et al (2012) found asymmetries between the effects of inputs to synapses on basal dendrites depending on whether they were near to or far from the soma, and that could provide a mechanism for dendritespecific contextual modulation (Mel, personal communication).

Neuronal signals can also be amplified indirectly by disinhibition, i.e. by reducing the activity of inhibitory neurons that suppress pyramidal cell spiking. Optogenetic studies have shown that under natural conditions the output of Layer $2 / 3$ pyramidal cells is amplified when the activity of a specific class of inhibitory interneurons is reduced (Atallah et al., 2012). This class is referred to as PV interneurons, because they express parvalbumin, a low-weight protein involved in various physiological processes, including neuronal signalling. They have been identified with chandelier 
and fast-spiking basket cells, which are local-circuit inhibitory interneurons with axonal arbors on somatic and perisomatic parts of nearby pyramidal cells. Given that PV interneurons are implicated in both divisive and multiplicative functions, we now need more information on the sources of their inputs. Their excitatory inputs are likely to include those from Layer 6 pyramidal cells, because activating them suppresses visually evoked activity in pyramidal neurons in Layer $2 / 3$ of the same cortical column (Olsen et al., 2012). Output from Layer 2/3 could therefore be amplified by suppressing Layer 6 pyramidal cells. A major task for the future is therefore to discover more about their excitatory and inhibitory inputs.

Ayaz and Chance (2009) used evidence from computational modelling to argue that inhibitory mechanisms can also be used in a more complex way to provide a form of multiplicative input gain modulation. This requires control of the level of noisy background input to the cell so that excitatory and inhibitory inputs are approximately balanced. Such a mechanism may therefore contribute to some of the amplifying effects reviewed above, but, even if it does, that is not evidence against the more direct mechanisms that we emphasize.

\subsection{Modulation that suppresses responses to $R F$ input}

Inhibition plays an important role in shaping RF selectivity, but, in addition to those inhibitory processes, responses to RF input are often suppressed when the stimulus to which the cell is tuned is surrounded by similar stimuli (e.g. Allman, Miezin and McGuinness, 1985; Heeger, 1992; Simoncelli and Schwartz, 1999). This is a much-studied example of suppressive modulation within the cortex, and has been compared directly with effects observed psychophysically (e.g. Kapadia, et al., 1995). Surround suppression increases coding efficiency by using predictions to suppress signals that have such high probability that they transmit little or no information. The suppression of selfgenerated signals, which occurs in several modalities, is another process of wide generality that also minimizes signals of such high probability that they transmit little or no information (Brown, et al., 2013).

Suppression is crucial to divisive normalization which has been described as a canonical neural computation because it may underlie so many different operations. These range from the formation of efficient codes by the reduction of redundancy in sensory systems to invariant object recognition, selective attention, and value encoding (e.g. Carandini and Heeger, 2012; Kouh and Poggio, 2008; Schwartz and Simoncelli, 2001). The classic form of divisive normalization implies suppression because the signals used to compute the numerator in the division are a subset of those used to compute the denominator. Input normalization flexibly adapts the dynamic range of pyramidal cell activity so that it remains sensitive to weak inputs without saturating in response to stronger inputs (Pouille et al., 2009).

Effects predicted by divisive normalization have been observed in many cortical areas of many species, e.g. V1, V2, MT, V4, inferotemporal cortex, auditory and olfactory cortex, multisensory areas, and areas where value is encoded such as monkey lateral intraparietal cortex (Carandini and Heeger, 2012, Louie and Glimcher, 2014). Although the effects and mechanisms of divisive normalization differ in detail across different areas there are also strong similarities, suggesting that they implement a common logic. It has been argued that normalization explains: why multisensory neurons weight each of their various inputs by the strength of the evidence that they provide; why multisensory enhancement decreases with stimulus intensity; and why a stimulus in a non-preferred modality can produce spiking when presented by itself but reduce spike rate when presented together with a stimulus of a preferred modality (Carandini and Heeger, 2012). Divisive normalization involves both feedforward and feedback inhibition, with shunting inhibition playing a major role (Shushruth et al., 2009). Louie, et al (2014) report many findings from an abstract computational model showing how temporal aspects of divisive normalization can explain many phenomena of context-sensitive value encoding in frontal and parietal cortices. Such extensions strengthen the claim that divisive normalization, in some form or other, is a canonical neural computation. No 
particular theory of divisive normalization is generally accepted yet, however. We must therefore make clear that our emphasis here is upon the many well-established suppressive phenomena upon which those theories are built. Exactly how those phenomena are best interpreted remains an open and important issue.

Suppressive modulation requires inhibitory mechanisms, and several classes of inhibitory neuron have been distinguished. Two are of particular importance here. One is referred to as PV interneurons as discussed in the preceding section. The other is referred to as SOM interneurons because they express the neuropeptide somatostatin. It includes Martinotti cells, which form axonal arbors on the tuft dendrites of neighbouring pyramidal cells. They are involved in the regulation of a wide range of processes (Martel et al., 2012). Neurons of both classes are widely distributed throughout all regions of mammalian cortex, and together they comprise a substantial proportion of all inhibitory interneurons (Martel et al., 2012; Rudy et al., 2011).

The roles of these two classes of interneuron in contextual modulation are now being revealed by optogenetic studies using both anesthetised and awake animals. They show that different classes of inhibitory interneuron provide mechanisms for different forms of contextual suppression. For example, SOM interneurons contribute to surround suppression. Selectively reducing their activity reduced surround suppression in Layer 2/3 pyramidal neurons by between 10 and 30\% (Adesnik et al., 2012). Furthermore, in accord with their putative role in surround suppression, SOM inhibitory interneurons target dendrites on nearby pyramidal cells with a wide range of orientation specificities (Wilson et al. 2012). SOM interneurons are but one of several mechanisms for surround suppression, however, which is also in part inherited from earlier stages of visual processing. Some other forms of modulatory suppression are exerted by PV interneurons. Combining optogenetic stimulation with in vivo two-photon imaging in mouse visual cortex Wilson et al. (2012) found that activating PV interneurons divisively reduced the slope of a Gaussian function fitted to the spiking of nearby pyramidal cells while leaving the spiking threshold, or offset, unchanged. In contrast to that, activating SOM interneurons had no effect on slope, but it did increase the spiking threshold and sharpen orientation selectivity. Other studies have found similar effects. For example, Atallah, et al. (2012) found that optogenetically activating PV interneurons decreased pyramidal cell activity divisively by a factor of 1.4 and subtracted a constant amount. Another optogenetic study found that activating PV inhibitory interneurons sharpened the orientation tuning and direction selectivity of the nearby pyramidal cells to which they were directly connected (Lee, et al., 2012b). In contrast to the findings of Wilson et al. (2012), the sharpening was specifically due to PV interneurons because activating SOM and VIP inhibitory interneurons had no such effect. Lee, et al. (2012b) showed the relevance of these effects to perceptual discrimination as measured psychophysically by activating PV interneurons in awake mice. They found that the improvement that this produced in orientation discrimination mirrored the sharpened V1 orientation tuning as measured electrophysiologically. Thus, PV interneurons play a central role in controlling the amplitude and precision of response to sensory input (Sohal et al, 2009; Cardin et al., 2009).

In sum, it is clear that suppressive modulation is widely distributed across mammalian cortex, and is implemented by various mechanisms that operate interactively within and across multiple levels of processing. It implements a general computational strategy in which highly probable or irrelevant signals are suppressed, thus increasing the salience of more informative or more relevant signals (Series, Lorenceau, \& Frégnac, 2003; Carandini and Heeger, 2012; Louie and Glimcher, 2014).

\subsection{Modulation that synchronizes responses to $R F$ input}

Until recent decades, studies of the temporal relations between the activities of different cortical neurons were usually carried out to examine causal relationships. Synchrony was then interpreted as evidence for input from a common source. Recent studies have been more concerned with the hypothesis that synchronized oscillations serve as a signal for dynamic grouping, however. It is on 
those that we focus here. Conceptual and psychological aspects of dynamic grouping are discussed in Section 4.3. This section focuses on neurobiological studies.

As there is much evidence for the hypothesis that dynamic grouping depends upon synchronized oscillations it has been much reviewed and debated (e.g. Singer, 1999; Shadlen and Movshon, 1999; Uhlhaas, et al., 2009; von der Malsburg, Phillips and Singer, 2010; Vinck, Womelsdorf and Fries, 2013). There is also evidence for other grouping strategies, however. For example, when macaques were viewing a display of Gabor elements arranged as in the contour integration paradigm, some V1 neurons increased their firing rates when the element to which they were selectively sensitive was part of the contour (Bauer and Heinze, 2002). This suggests that figures may be distinguished from ground by amplified firing rates. The effect started with a latency of about $150 \mathrm{~ms}$, however, and macaques require stimulus-mask asynchronies of only about 30 to $60 \mathrm{~ms}$ to detect a contour within a complex background (Mandon and Kreiter, 2005). This suggests that there is more to dynamic grouping than amplification of firing rates as measured by Bauer and Heinze. More recent results suggest that amplification and synchronization may both be involved in grouping. For example, Ramalingam et al. (2013) found that in awake behaving macaques perceptual grouping increased the synchronization of LFPs in V1, whereas perceptual segregation lowered synchronization. These effects were greatest when the feedforward drive was present but weak. However, Ramalingam et al. (2013) also found that V1 cells have higher firing rates when the element to which they were selectively sensitive was part of a contour. Amplification increased with contour length, and occurred with a latency of about $100 \mathrm{~ms}$ following stimulus onset. An important difference between the context-sensitive LFP and spike-rate measures used in those studies was that there were substantial task-dependent LFP differences prior to stimulus onset, but there were no contextual effects on the spiking of superficial neurons until after stimulus onset. This is in accord with our assumption that context is usually insufficient to generate output signals by itself, even though it often has strong effects given a signal to modulate.

Many studies have suggested that oscillations at gamma frequencies of the feedforward signals may be particularly relevant to the transient formation of neuronal groups or assemblies. Gamma rhythms have been observed in many different species, and their synchronization within and across local neuronal groups has been observed both within and between many different brain regions (Fries, Nikolic and Singer, 2007; Vinck, Womelsdorf and Fries, 2013; von der Malsburg, Phillips and Singer, 2010). Gamma-band synchronization is stronger for salient stimuli, and increases with perceptual grouping and spatial integrity (Fries et al, 2001a; Lima et al., 2010; Zhou, Bernard and Bonds, 2008). It is particularly strong in superficial layers and during active, awake states (e.g. Fries et al., 2001b). Gamma rhythms may also help separate relevant signals from noise because noise is not synchronized to the gamma cycle (Buzsáki, 2006).

Though some studies have failed to support the view that synchronization and desynchronization are used to signal grouping and segregation (e.g. Lamme and Spekreijse, 1998), many do. For example, when late-stage LFPs in monkey V1 were studied, contours separating figure from ground desynchronized non-rhythmic activity as well as rhythmic activity, providing that it was within the gamma frequency band (Gail, Brinksmeyer, and Eckhorn, 2000). The gamma cycle is well-suited to perceptual organization because information is transmitted rapidly by the latency of the first spike or burst, and updating can occur every 25 milliseconds or so (Fries, Nikolic and Singer, 2007). There is also evidence that different subsets of events within a gamma cycle may be distinguished by their phase relative to the cycle (Vinck, Womelsdorf and Fries, 2013), but, whether that is so or not, gamma rhythms and their synchronization seem to play a major role in dynamic grouping.

Some forms of dynamic grouping require attention, and it has been shown that attention strongly enhances gamma-band synchronization both within (Fries et al., 2001b) and between cortical regions (Gregoriou et al., 2009). Furthermore, in addition to any role that it has in grouping, attention may also affect amplification and suppression via its effect on the synchrony of inhibitory interneuron activities. Computational modelling suggests that interneuron synchrony could affect the slope of the 
function relating the postsynaptic neuron's output to its RF input (Tiesinga, Fellous and Sejnowski, 2008). This could mediate the changes in firing rate that are seen in conjunction with selective attention, and this form of gain modulation increases or decreases the overall strength of the neuron's response while preserving RF stimulus specificity. This computational modelling also shows that the timescale of the inhibitory conductances involved are such that modulation is best achieved for oscillations in the low gamma frequency range.

There is now broad agreement that PV inhibitory interneurons provide a core mechanism for generating gamma rhythms. Optogenetic studies show that activating them strongly enhances gamma-band oscillations in the LFP (Cardin et al., 2009), suggesting that they provide a sufficient mechanism for gamma oscillations. Furthermore, when gamma oscillations are induced by feedforward activation, they are suppressed by the inhibition of PV interneurons (Sohal et al., 2009), suggesting that PV activation is not only sufficient but necessary.

There are grounds for supposing that mean activity levels, synchronization, and contextual modulation are all tightly linked. Computational modelling suggests that, as activity levels increase, smaller membrane time constants promote synchronous gain in the network, i.e. cells become more sensitive to temporal coincidences in their synaptic inputs, thus responding more to synchronous than to asynchronous inputs (Chawla, Lumer and Friston, 1999).

\subsection{Overview of the neurobiological evidence}

There is clear neurobiological evidence for modulatory processes of amplification, suppression, and synchronization in many different cortical regions of many different species. There are several theoretical interpretations of these findings, varying, for example, in the extent to which they emphasize amplification and suppression, but common to all is that context, broadly conceived, modulates responses to feedforward or driving input (Muckli and Petro, 2013). Though much remains to be discovered concerning the mechanisms by which it does so (Silver, 2010), some major candidates are now becoming clear. One of the most important is apical amplification, either via BAC firing or via other mechanisms. Its regulation via inhibitory interneurons is also crucial. Apical amplification provides pyramidal cells with a mechanism by which they can modulate the responses of other pyramidal cells directly (Larkum, 2013; Major, Larkum, and Schiller, 213, Palmer et al., 2014). This may have major advantages over doing so indirectly via inhibitory interneurons, which modelling shows to be an alternative possibility (Ayaz and Chance, 2009). As current evidence suggests that apical amplification is common to pyramidal cells in many different cortical regions, it is likely to have far reaching implications for our understanding of cortical computation in general.

The evidence reviewed above suggests that fast-spiking PV inhibitory interneurons contribute to all three forms of modulation: activating them implements some forms of suppression; inhibiting them implements indirect forms of amplification; and appropriately synchronizing the gamma rhythms that they generate implements some forms of dynamic grouping. SOM interneurons have been shown to play an important role in surround suppression. As they target the dendrites of the apical tuft that also receive much other contextual input, however, we expect that they will be found to have a much broader significance. Furthermore, if the functions of somatic, basal, apical and tuft compartments differ as implied by the hypothesis of apical amplification, then the effects of inhibitory regulation will depend on which compartment receives the inhibition. We therefore expect such inhibitory effects, and any disinhibitory mechanisms that are associated with them, to be a major focus of research in the coming decades.

Another major focus will be the distinct functional roles of synchronization within the gamma and beta frequency bands. This is of particular relevance to feedback modulation because it has been shown that across many different cortical regions feedforward signalling involves synchronization at gamma frequencies and that feedback modulates feedforward signalling via synchronization at beta frequencies (Bastos et al, 2015). 


\section{Perceptual and higher cognitive functions that depend upon contextual modulation}

The aim of this section is to relate contextual modulation as observed at the neuronal level to psychological and computational studies of cognition. It has been implicated in a wide variety of psychological functions (e.g. Lamme, 2004; Fries, Nikolic and Singer, 2007; von der Malsburg, Phillips and Singer, 2010). Here we summarize the evidence for some of them including: contextual disambiguation; surround suppression and normalization in perception; Gestalt grouping; selective attention; sensory-motor coordination; the dynamic creation of transient assemblies in working memory; cognitive control; and the selective formation of longer-term memories. It is also possible that the dynamic formation of widely distributed but transient coalitions of mutually supportive activities by long-range gamma-synchronization is a neural correlate of sensory awareness and consciousness (Engel and Singer, 2001; Koch, 2004; Melloni, et al. 2007). Furthermore, apical amplification may be a major determinant of the level of consciousness reached by any given content at any given moment (Bachmann and Hudetz, 2014).

\subsection{Contextual disambiguation in perception}

A fundamental challenge faced by perceptual systems is that of interpreting ambiguous signals (Rust and Stocker, 2010). This ambiguity can be reduced by amplifying interpretations that are predicted by contextual inputs from other processors in the same or higher regions. Local competitive interactions would then lead to suppressive effects on alternative interpretations. In natural images this contextual guidance is usually sufficient to resolve all or most of the ambiguity. In the limit, contextual disambiguation can even support the 'identification' of objects in degraded images when they are effectively represented only by a single pixel (Torralba, 2009). Contextual disambiguation by amplifying modulation increases the salience of signals as their probability given the context increases. In contrast to divisive normalization, it does not require the use of any pooled normalizing average, and it is well-suited to cases where the context makes weaker, less dominant, interpretations the better alternative. Well-established effects of contextual disambiguation range widely across modalities and levels of processing. Here we give a few examples of its use to facilitate signal detection, to solve the aperture problem in motion perception, to produce coherent objectdescriptions, to facilitate object-recognition within scenes, and to guide multi-stable perception.

The facilitation of low-contrast edge detection by collinear flankers is a well-established example of the use of specific contexts to resolve the ambiguity between signal and noise (Polat and Sagi, 1993). Much has now been learned about facilitation by flankers, including evidence that it involves amplifying modulation (Chen and Tyler, 2008; Huang, Hess and Dakin, 2006; Maehara, Huang and Hess, 2010), can be cancelled by non-collinear flanks (Solomon and Morgan, 2000); depends upon input from other parts of the adjacent surround (Mareschal and Clifford, 2013), and is itself modulated by attention (Ramalingam et al., 2013). Psychophysical studies show that detection is improved when the flankers precede the target, or are presented simultaneously with it, but not when the target precedes the flankers (Polat and Sagi, 2006). This provides further evidence that the effect of flankers arises from long-range intracortical facilitation, rather than from feedforward summation.

Motion perception also requires contextual disambiguation. The motion of an edge extending beyond the distal RF of an orientation sensitive cell in V1 is inherently ambiguous because the temporal changes measured locally could be due to many different motions of the object as a whole. It has been demonstrated computationally that this ambiguity can be resolved by using feedback from higher regions, such as MT, to amplify coherent interpretations (Bayerl and Neumann, 2004). The rationale for this is that, as MT neurons receive measures from a larger spatial range than V1 neurons, they could use that larger context to guide the local interpretations in V1 toward a coherent interpretation of the object motion as a whole. Feedback could thus enhance local signals compatible with predictions from that broader context. As this will have the effect of amplifying those activations in V1 that match MT's predictions it would give them a strong advantage in the local 
competitions to which they are subject. It would not be necessary for this modulatory feedback to produce any output from V1 by itself, however (Bayerl and Neumann, 2004). This core computational logic can solve the aperture problem when all the local motion signals are ambiguous (Perrinet and Masson, 2012); it can resolve ambiguities in static form perception (Neumann and Sepp, 1999); it performs well when applied to natural images (Bayerl and Neumann, 2004); and a neuronal implementation could in principle perform rapidly (Bayerl and Neumann, 2007).

A central role for contextual disambiguation in computer vision was shown long ago by using language-like 'picture-grammars' to interpret line drawings of opaque polyhedra (Clowes, 1971). Clowes showed formally how local ambiguity can be reduced by using compatibility constraints to select interpretations of local features so that they form a coherent whole. For further discussion of the relevance of Clowes' computational studies of contextual constraints to contextual modulation see Phillips, von der Malsburg and Singer (2010). For a state-of-the-art example of a computer vision system that uses contextual disambiguation for natural scene interpretation see Rasiwasia and Vasconcelos (2012). For a biologically based neurocomputational study showing the effectiveness of context-sensitive disambiguation at the level of local feature processing see Krüger and Wörgötter (2005).

Many studies of object recognition have shown that there are strong biases toward interpretations that are more probable given the context (Bar, 2004; Torralba, 2009; Oliva and Torralba, 2007). Effects of these contextual biases range from facilitation of object-naming at the subordinate level (Davenport and Potter, 2004) to the rapid detection of super-ordinate object categories such as animals (Fize, Cauchoix and Fabre-Thorpe, 2011). Furthermore, the latter study also showed that the facilitation of object recognition by learned associations between object and background scene is very similar in humans and macaques. This facilitation seems to operate pre-attentively (Munneke, Brentari, and Peelen, 2013).

The phenomena of multistability bring the local ambiguities that are inherent in image interpretation into direct experience. When perception switches between alternative interpretations it usually does so as a whole, implying that all the distinct decisions that this involves are coordinated by some form of contextual modulation that maximizes coherence over the figure as a whole (Klemm, Li and Hernandez, 2000). Studies of multistable perception provide much evidence for a strong bias towards those interpretations that are more probable given the context (Schwartz et al., 2012). A recent review of such studies concludes that spatial, temporal, and cross-modal contexts can all be effective in this way (Klink, van Wezel and van Ee, 2012).

Contextual disambiguation is also central to one of the best established and most influential theories of human word and sentence comprehension (Landauer and Dumais, 1997), and to ConceptNet, which is a software package for natural language processing that achieves success in many textual reasoning tasks by applying context-oriented inference to a large real-world knowledge base (Liu and Singh, 2004).

\subsection{Surround suppression and divisive normalization}

If the amplification of signals as their probability given the context increases were taken to the limit then that would amplify signals conveying little or no information. Therefore, at high levels of signal probability given the context, signal strength should not be increased but should be reduced, as in surround suppression. The perceptual consequences of surround suppression have been muchstudied, and provide a classic case where the relationship between the psychophysical and neuronal measures has been shown to be very close (e.g. Kapadia, et al., 1995). For examples of the effects of surround suppression on pop-out in visual search, and its relation to dynamic grouping see Watt and Phillips (2000).

Divisive normalization has been related to a wide variety of psychophysical phenomena (Carandini and Heeger, 2012), including those that are non-local as well as those that are local, such as adaptation by cortical contrast gain-control (Ohzawa, Sclar and Freeman, 1985). Many 
behavioural and phenomenological consequences of the suppression of self-generated signals are reviewed by Brown et al (2013), who also show how such findings can be interpreted from the perspective of the theory of free energy reduction.

\subsection{Gestalt grouping}

The computational necessity and psychological significance of processes that organize distinct 'parts' into coherent 'wholes' has been so well-established for so long that they are at the centre of a major school of psychological thought, i.e. Gestalt psychology. Though often treated as of relevance mainly to perception this perspective has made major contributions to many other areas as well, including those of memory, thought, and abnormal psychology (Silverstein and Uhlhaas, 2004). As a review of that whole perspective is not feasible, our aim here is simply to show that Gestalt grouping shares basic functions and mechanisms with other forms of contextual modulation.

The dynamic grouping of neuronal activities, both within and across cortical areas, is widely thought to be one of the basic functions of contextual modulation in visual cortex (e.g. Lamme, 2004; von der Malsburg, Phillips and Singer, 2010). Signals must be dynamically grouped into coherent sub-sets at each level of processing so that they can have appropriate effects at the sites to which they project. Storing sensory input as a single whole would be of little use because the 'curseof-dimensionality' would then prevent learning (Bellman, 1961; Phillips, 2012). Therefore it is necessary to group the sensory input into coherent subsets, and criteria by which this grouping may be achieved have long been studied by Gestalt psychologists and many others. Though this is often referred to as 'the binding problem' that terminology obscures crucial distinctions. Here we use the general term 'grouping' to refer to the organization of data into subsets relative to a particular operations performed upon them. The grouping implicit in RF selectivity is specified prior to knowledge of the particular data set that is to be organized into groups; therefore we refer to it as 'pre-specified grouping'. This contrasts with 'dynamic grouping', which requires the current data to be known. Dynamic grouping is assumed to be pre-attentive when it occurs rapidly and in parallel across the visual field, and attentive when it is slower, serial, and interfered with by other demands on attention (Phillips, von der Malsburg and Singer, 2010; Watt and Phillips, 2000).

Though both forms of dynamic grouping differ from amplifying and suppressive modulation they have important commonalities with them. First, they must organize signals into coherent subsets without corrupting the information that those signals transmit. Second, evidence reviewed in Section 3 indicates that dynamic grouping depends on some of the same mechanisms as those involved in amplification and suppression. Third, evidence reviewed in Section 5 below indicates that all three forms of contextual modulation tend to be impaired in schizophrenia-spectrum disorders. Note that though these three forms of contextual modulation are distinct they may not operate independently. It is likely that they operate interactively, with amplification depending upon grouping processes, for example (Sayim, Westheimer and Herzog, 2011).

Rigorous psychophysical comparisons between contour integration and flanker facilitation, a paradigmatic case of amplification, shows that they have similar but distinct mechanisms (Huang, Hess, and Dakin, 2006). Most of the evidence concerning those mechanisms comes from studies of non-human animals, however. It is therefore important to note that psychophysical studies by Mandon and Kreiter (2005) show that human performance in the contour integration paradigm is much the same as that in macaques.

Recently developed methods for analysing multi-electrode EEG recordings are now providing evidence that contour processing involves synchronized activity at various frequencies. For example, it has been shown that detection of the figure involves synchronization at gamma frequencies within early visual regions, whereas categorization of the orientation of the egg-shaped figure formed by the contour involves synchronization at beta frequencies across a network that includes frontal and parietal regions (Castellano et al., 2014). 
If synchronization at gamma frequencies around $40 \mathrm{~Hz}$ is a signal for Gestalt grouping then neural signals that are synchronized to about 20-30 ms should be grouped and those that occur with longer asynchronies should be segregated. Furthermore, it has been hypothesized that temporal precision to within $20-30 \mathrm{~ms}$ has special significance in the neocortex because that is both the duration of the burst produced by apical amplification and the coincidence time window that leads to the burst (Larkum, Senn and Lüscher, 2004). This issue has been directly investigated by psychophysical studies of the effects of small differences in onset times on figure-ground segregation in the contour integration paradigm (Hancock and Phillips 2004; Hancock et al., 2008). Most, but not all, of the people tested could reliably detect contours hidden within the random background provided that their onset times differed by at least 20 - $30 \mathrm{~ms}$. Detection was just as good when the figure followed the ground as when it preceded it, which is counter-intuitive because when the figure was presented first it was presented without any interfering background. This shows that whether events were seen as being grouped or segregated depended upon the amount of asynchrony between events, not upon their order. Furthermore, the effects of asynchrony were independent of the spatial cues to contour detectability. Thus, the width of the time window within which events are seen as being grouped is the same as that of a gamma cycle and as that within which basal and apical depolarisations must co-occur for apical amplification to operate.

\subsection{Perceptual invariance and adaptive modification of RF selectivity}

Contextual modulation also contributes to perceptual invariance and guides adaptive changes of RF selectivity. Here we give only a few examples. First, divisive normalization computes outputs that are sensitive to relevant but not irrelevant stimulus dimensions because in discarding information about mean levels of activity it computes representations that are invariant across changes in that mean (Carandini and Heeger, 2012). Therefore it can make a major contribution to solving core problems of invariant object recognition (DiCarlo, Zoccolan and Rust, 2012). Second, amplifying modulation contributes to invariant object recognition by making it context-sensitive. Third, predictive relationships that are relevant to each particular brain region can be discovered by adjusting the strengths of the synapses that mediate RF and contextual inputs so as to amplify signals that are statistically related to the distinctive set of contextual inputs that neurons in each particular brain region receive (Kay, Floreano and Phillips, 1998). For example, selective sensitivity to invariant object properties could be acquired in inferotemporal areas where structural descriptions of the image are related to contextual inputs arising from properties of objects that are invariant across different sensori-motor interactions with them. In contrast to that, sensitivity to variables predictive of the details of specific sensori-motor interaction could be acquired in posterior parietal areas where the feedforward signals are interpreted in a sensori-motor context. Fourth, selective sensitivity to motion signals that are locally unambiguous could be acquired as a consequence of using feedback signals to resolve ambiguity (Perrinet and Masson, 2012). Finally, visual responses with substantial invariance across space can also be produced by the modulatory effects of attention (Salinas, 2009).

\subsection{Selective attention and cognitive control}

As attention enhances selected signals and suppresses irrelevant signals it is one of the main cognitive functions that depends on contextual modulation (Thiele, et al., 2009). Furthermore, as also noted above, the processes of contextual modulation depend upon attention. The biasedcompetition theory (Desimone and Duncan, 1995) and the normalization theory of attention (Reynolds and Heeger, 2009) both show how many well-established properties of attention can be explained by implementing attention as a multiplicative amplification combined with divisive normalization. Note, however, that even in models in which attention is described as 'additive' (Thiele, et al., 2009), multiplicative effects are assumed at sub-threshold levels. Attention can therefore be seen as a major source of the contextual input that modulates neuronal response to RF input. This is in harmony with recent developments of the divisive normalization theory (Carandini 
and Heeger, 2012). The sources of contextual input are of course different in these different uses, but their effects on local processor activity are essentially the same, thus allowing them to share common local circuit and intracellular mechanisms.

Attentional influences play a major role in the theory of free energy reduction and predictive processing (Feldman and Friston, 2010). This offers a formal way to understand contextual modulation in a global sense. It can be separated into first and second order aspects of the models used by the brain to predict sensory information. These generative models necessarily have two non-linear or modulatory aspects that call upon gain control. First order contextual affects are a necessary part of any non-linear model that reflects the interaction among causes or objects in the environment producing sensory data, such as changes in the two dimensional view of a three dimensional object when it is rotated. Second order contextual effects relate to the second order statistics of sensory information, which are referred to as 'precision'. A simple example here could be the loss of precise visual input due to partial visual occlusion of one object by another. The brain has to predict both first and second order statistics and calls upon modulatory mechanisms for both.

In predictive coding theory attention is associated with updating the precision or confidence in prediction errors, thus relating contextual modulation to the encoding of uncertainty or second order context. Physiologically, this corresponds to the contextualization of neuronal message passing as described above. Effectively, attention selects specified processing channels by increasing their precision or attentional gain while suppressing others. There are a number of alternative architectures for predictive coding of this sort. In some proposed architectures feedforward pathways transmit prediction errors (e.g. Friston, 2010). In others current inferences are signalled by feedforward inter-regional pathways with the processing of prediction error being performed within local cortical circuits (Spratling, 2008, Spratling, De Meyer and Kompass, 2009). A computational model performing perceptual inference in essentially that way has been shown to develop both driving and modulatory interactions between local processors, and to account for many aspects of selective attention, surround suppression, flanker facilitation, and contour integration (Spratling, 2014). Though not in principle dependent on it, the top-down amplifying modulation in those computational models could be sent to the apical dendrites of the earlier population (Spratling, personal communication), and some earlier models were explicitly based on the possibility of separate apical and basal integration zones (Spratling and Johnson, 2004, 2006; De Meyer and Spratling, 2009). These possible architectures are not mutually exclusive because some feedforward pathways could signal errors, while others signal inferences. A problem for both kinds of model is that pyramidal cells that signal errors, either within or between regions, have not yet been clearly shown to exist. This is not problem for the theory of coherent infomax that is discussed in Section 6.2 because that does not rely upon the signalling of prediction errors. A problem for that theory, however, is that, being formalized at the abstract level of computational theory, it leaves many implementational issues unresolved.

Cognitive control, as in the Stroop task for example, is a classic case of the need to selectively use information that is currently relevant, and ignore that which is irrelevant. Fenton and colleagues have developed a rodent version of this task that can be used to study several crucial neurobiological and behavioural aspects of cognitive control (Wesierska, Dockery, and Fenton, 2005; O'Reilly et al., 2014). Rodents are exposed to two competing streams of information, only one of which must be used to avoid shock. They are placed on a slowly rotating arena with a shock zone that is defined within the room coordinates. Because only the room cues provide relevant information for avoiding the shock, they must not associate shock with locations defined relative to the rotating arena. Fenton, et al., found that normal laboratory rodents quickly learned this task, reaching asymptotic performance within three 10-min trials. Furthermore, they showed that the rat's ability to selectively use the relevant spatial framework was impaired by unilateral lesions of the dorsal hippocampus. A later study demonstrated that injection of tetrodotoxin (TTX) induced coactivation of previously 
weakly associated cells. This added noise to the previously well-coordinated cellular activity, and occurred largely within the gamma band (Olypher, Klement, \& Fenton, 2006).

\subsection{Flexible selection and coordination of actions}

Contextual modulation has a major role in coordinating flexible mappings from perception to action. Friston (2010) reviews much evidence suggesting that predictive error-correcting processes, and the control of precision on which they depend, play a central role in motor control as well as in perception. In the case of motor control these processes can be seen as progressively reducing the difference between current and intended states.

Computational models show how contextual modulation can be used to rapidly and flexibly adapt actions to current conditions by selecting one of several possible mappings from sensory stimuli to motor actions, (Salinas, 2004). If somebody suddenly touches your hand, your reaction will depend on who is touching your hand and why. Clearly, the impact of a stimulus on your behavior will depend on other concurrent stimuli, short and long-term memories, current motivations and goals, etc. The neural network model of Salinas (2004) shows how mappings with the required flexibility can be achieved by using modulation to select one of several possible mappings from sensory input to motor action, depending on the context. That model uses essentially the same form of modulation that we hypothesize to be used, in various ways, throughout the cortex.

The breadth of its potential relevance is a hall-mark of contextual modulation. For example, it has been argued that perceptuomotor trajectories emerge and are maintained within circular causal webs (Cisek and Kalaska, 2010). Within those webs estimated uncertainty and adjustments of gain enable the context-sensitive selection and coordination of actions. Cisek and Kalaska propose that this is done by biasing competition between the multiple parallel streams of processing that specify the parameters of alternative possible actions using modulatory interactions that are essentially the same as those proposed above. Thus, their distinction between action specification and action selection is essentially equivalent to our distinction between driving and modulatory interactions.

\section{Malfunctions of contextual modulation in schizophrenia spectrum disorders}

This section outlines ways in which studies of schizophrenia have cast light on the neuronal mechanisms of contextual modulation and on its role in mental life. There is evidence that impaired sensitivity to context plays a major role in producing the cognitive deficits and delusional experiences seen in schizophrenia (Phillips and Silverstein, 2013). Mitchell et al. (2013) argue that schizophrenia may be more understandable at the level of core pathophysiology than at the levels of aetiology and symptomatology. Here we review evidence suggesting that its core pathophysiology involves contextual modulation. Impaired contextual modulation has been observed in several neuropsychiatric and genetic conditions (e.g., Silverstein, 2010), but most prominently in schizophrenia spectrum disorders. In the sections below we outline some of the evidence for changes in contextual disambiguation, surround suppression, normalization, Gestalt grouping, and higher cognitive functions such as selective attention, cognitive control, and the sense of agency. We also briefly summarize evidence that they involve altered functioning at NMDARs and inhibitory interneurons. As both perception and higher cognitive functions are impaired in these disorders they provide evidence on the widespread role of contextual modulation in mental life.

The evidence reviewed here is in broad agreement with the computational model of psychosis presented by Adams, et al (2013). They present strong grounds for the view that many schizophrenic abnormalities, including delusional and hallucinatory inferences, arise from aberrant encoding of precision, i.e. from impairments of what we refer to as contextual modulation. Related impairments could also play a central role in autism, as that may also involve an aberrant setting of precision (Lawson, Rees, and Friston, 2014; Quattrocki and Friston, 2014). It seems likely that autistic disorders differ from schizophrenic disorders because they involve serious malfunctions of contextual modulation much earlier in the course of development. If so, future comparisons between 
these two apparently very different classes of disorder may cast much light on the role of contextual modulation in cognitive development.

\subsection{Contextual disambiguation}

There is clear evidence that schizophrenia involves abnormalities of contextual disambiguation in perception. Some of this comes from psychophysical studies of flanker effects, where there is in effect an ambiguity between signal and noise. Compared to controls, patients demonstrate reduced facilitatory effects of collinear flankers on detection of a target at short target-flanker distances (Kéri et al., 2005). They also show lack of attentional modulation of this effect (Kéri et al., 2009), which is a further example of the reduced effects of modulatory amplification. Such patients also need more of the contours to be present to identify objects, so that is further evidence of impaired disambiguation (Doniger, et al., 2002). At higher cognitive levels, sentence comprehension is a paradigmatic example of cases where contextual disambiguation is crucial, and there too, schizophrenia patients demonstrate weakened effects of context (Andreou et al., 2009).

\subsection{Surround suppression and divisive normalization}

Several failures of surround suppression have been observed in schizophrenia. For example, patients have demonstrated weaker suppression for contrast (Dakin, Carlin and Hemsley, 2005; Tibber et al., 2013), and size (Uhlhaas et al., 2005, 2006; Silverstein et al., 2013), with mixed evidence on orientation (Schallmo, Sponheim, and Olman, 2013; Tibber et al., 2013; Yoon et al., 2009, 2010). There is negative evidence in the case of luminance, the earliest processed of the dimensions studied, and that suggests a cortical locus for reduced surround suppression (Tibber et al., 2013). Other results, such as reduced crowding effects and some findings on poor contour detection have also been attributed to weaker surround inhibition (Robol et al., 2013). In contrast to these findings is evidence that suppression of motion information in schizophrenia may in some cases be increased compared to healthy controls (Chen, Norton and Ongur, 2008). Overall, however, there is ample evidence that surround suppression is often impaired in schizophrenia (Yoon et al, 2013).

Failures of normalization have been observed in sensory gating paradigms. For example, numerous studies have demonstrated reduced pre-pulse inhibition (Braff, Geyer and Swerdlow, 2001), and reduced P50 suppression following an initial auditory burst (Patterson et al., 2008). Multiple studies have also observed reduced pre-attentive inhibition of repeated materials in auditory mismatch negativity (MMN) paradigms (see Näätänen \& Kähkönen, 2009 for review), and preliminarily in visual MMN (Urban et al., 2008). Taken together, this evidence, from multiple domains of sensation and perception and from multiple paradigms supports the view that processes of modulatory suppression are abnormal in schizophrenia.

\subsection{Gestalt grouping}

Dynamic grouping failures have been repeatedly observed in schizophrenia in the form of perceptual organization impairments. This has now been demonstrated in over 50 studies (reviewed in Uhlhaas

$\&$ Silverstein, 2005; Silverstein \& Keane, 2011). These findings are not due to medication effects, or to generalized performance deficits: in cases where grouping of targets and distracters interferes with task accuracy, schizophrenia patients demonstrate greater accuracy than controls (e.g., Place \& Gilmore, 1980; Silverstein et al., 2013; Uhlhaas et al., 2006). These perceptual organization impairments are considered examples of failures of dynamic grouping because they are demonstrated most clearly when non-contiguous elements need to be combined and where prepotent stimulus structure is weak. Performance is normal when perceptual organization can be easily achieved by pre-specified feature hierarchies. Perceptual organization impairments in schizophrenia have been demonstrated using fMRI (showing reduced activation in occipital areas regions sensitive to grouping but normal V1 activity; Silverstein et al., 2009), event related potentials such as closure negativity (Ncl; Butler et al., 2013), and EEG measures such as gamma power and gamma 
synchrony (reviewed in Uhlhaas \& Singer, 2010, 2013). A version of the contour integration paradigm in which figure-ground segregation is cued by onset asynchrony also shows that both schizophrenia and schizotypy are associated with substantially reduced temporal resolution at gamma frequencies (Hancock et al., 2008).

In addition to reduced grouping in vision, schizophrenia also provides examples of reduced organization in other domains, including thought and language, motor functioning, and consistency between affect and ideation (i.e., inappropriate affect). Importantly, abnormalities in these domains are significantly correlated with each other (Lindenmayer, Bernstein-Hyman, \& Grochowski, 1994; Walther et al., 2014), and with severity of perceptual organization impairments (reviewed in Phillips \& Silverstein, 2003; Uhlhaas \& Silverstein, 2005; Silverstein \& Keane, 2011). This all suggests that in schizophrenia there is a widespread failure in dynamic, context-sensitive, grouping.

\subsection{Selective attention, cognitive control, and the sense of agency}

Impaired selective attention is one of the most consistently demonstrated cognitive features of schizophrenia (e.g., Wishner \& Wahl, 1974; Ward et al., 1991; Tregellas et al., 2012), and can be considered prima facie evidence for reduced contextual modulation in schizophrenia in both perceptual and other functions. Further evidence comes from data on cognitive control, which is also considered a core feature of the disorder. A recent review of the large body of evidence on this issue (Lesh et al., 2011) noted that reduced cognitive control involves reductions in dendritic spines, reduced GABA release in PV interneurons, and reduced PFC-related gamma band activity - all of which are consistent with the evidence cited above. In addition, decreased cognitive control in schizophrenia is related to an increase in disorganized symptoms, mirroring the findings from studies of reduced perceptual organization in the disorder (Phillips \& Silverstein, 2003; Uhlhaas \& Silverstein, 2005; Silverstein \& Keane, 2011). These data from patients are supported by experimental studies in animals (O'Reilly, et al., 2014), including those demonstrating methods by which schizophrenia-related cognitive control impairments may be reduced (Lee et al., 2012a).

There are good grounds for proposing that an impaired sense of agency, e.g. as in thought insertion, arises from decontextualization, either via an impaired sense of ownership of one's own thoughts (Martin and Pacherie, 2013) or directly (Fletcher and Frith, 2009; Seeger, 2013). These altered states of conscious experience include distortions in body representations and a reduction in the coordinated representation of body parts and somatosensory signals (Graham et al., 2014). Thus psychotic experiences provide clear support for the hypothesis that contextual modulation plays a central role in the phenomenology of mental life. An explicit analysis of relations between such experiential phenomena and the core neurobiological mechanisms of contextual modulation reviewed in Section 3, including apical amplification, is therefore an important task for the future.

\subsection{NMDAR function and GABA-ergic activity are altered in schizophrenia}

Much evidence now suggests that schizophrenia is characterized by both reduced activity at NMDA receptors (Moghaddam \& Javitt, 2012; Kantrowitz and Javitt, 2010) and reduced activity in PV containing GABA-ergic neurons (Lewis et al., 2012). This leads to reduced context-sensitivity and reduced inhibition. Moreover, these disturbances have been linked to a variety of perceptual and cognitive failures in schizophrenia, including those in selective attention, context-sensitive amplification, some forms of dynamic grouping and segregation, and aspects of cognitive function that depend on synchronization of neural oscillations within the gamma band (Cobb et al, 1995; Pouille and Scanziani, 2001). Reduced GABA concentration in the occipital lobe in schizophrenia patients has also been linked to reduced orientation-specific surround suppression (Yoon et al., 2010).

We have reviewed evidence suggesting that PV interneurons play a major role in contextual modulation. In addition to that, Behrens and Sejnowski (2009) have reviewed evidence suggesting that dysregulation of PV interneurons in the developing cortex could explain the late onset of 
schizophrenic symptoms as well as the differences between the effects of brief and prolonged exposure to NMDA antagonists (Jentsch and Roth, 1999). Questions remain, however, as to exactly how PV interneurons are involved in the downstream effects of reduced activity at NMDARs (Gonzalez-Burgos \& Lewis, 2012).

Compelling evidence that NMDAR-hypofunction can produce schizophrenic symptoms comes from autoimmune anti-NMDAR encephalitis. This progressively reduces activity at NMDA receptors by capping and internalizing them (Hughes et al., 2010). Such patients present with symptoms that are so like schizophrenia that, prior to its further progression, schizophrenia is often the initial diagnosis. Our view of the role of NMDA receptors in contextual modulation therefore predicts that, when tested by rigorous psychophysical methods, it will be found to be impaired in such patients.

NMDAR and GABA-ergic malfunctions in schizophrenia are consistent with our emphasis upon apical amplification as a mechanism by which contextual inputs to apical tuft synapses amplify pyramidal cell output, but we know of no studies that directly implicate that mechanism in schizophrenia.

\section{Formal domain-free conceptions of contextual modulation and its long-term objectives}

Here we discuss contextual modulation at the abstract level of computational theory. The functions of contextual modulation as seen from each of three information-theoretic perspectives are considered. The first is that of a whole neural system operating within its ecological niche. The second is that of pyramidal cells, and their local circuit neurons, operating within the cortex as a whole. The third is from the perspective of a person's direct experience. Though different, the formal objectives related to each of those three perspectives seem to be fundamentally compatible.

Two aspects of biological function must be distinguished: 'function' as that for which something was selected, e.g. by natural selection; and 'function' as that which something contributes to the life of the individual organism of which it is a part (Godfrey-Smith, 1996). These two aspects are considered here from the perspectives of two computational theories, both of which propose a central role for contextual modulation. The first, discussed in Section 6.1, is the theory of free energy reduction (e.g. Friston, 2010). The second, discussed in Section 6.2, is the theory of 'coherent infomax' (e.g. Kay and Phillips, 2011). Both use information-theoretic notions to formulate hypotheses concerning the long-term objectives of neural systems. Section 6.3 then discusses their possible relevance to the directly experienced life of the individual.

\subsection{Staying viable by prediction error minimization}

It has been argued that life and mind share deep organizational principles, and that an understanding of mind is continuous with an understanding of life (Clark, 2001). That would not do much to help us understand cognition's fundamental goals, however, if, other than aiding survival and reproduction, cognition is nothing more than a large rag-bag of ploys and tricks providing specialized solutions to specialized problems. Intuitively, however, cognitive processes do seem to have something in common, i.e. their reliance upon adequately transduced information from past and present to guide activity in ways that are more flexible than direct stimulus-response links would allow. If so, what common neurally computable quantities could guide such fast flexible reorganizations, and how could that involve contextual modulation?

One possible quantity is prediction error (e.g. Friston, 2010). Minimizing prediction error, if this view is correct, is the common goal to which neural re-organization is directed, in both the shortterm and the long-term. Prediction error minimization (PEM) expresses several goals, including homeostasis, empirical variational forms of Bayesian inference, and the increase of adaptive fitness (Friston, 2010, 2013; Friston and Stephan, 2007). 
This theory offers a conception of neuronal dynamics that is relevant to both perception and action. Making inferences by reducing prediction errors is hypothesized to use generative models to both interpret sensory input and to produce actions that the models prescribe. Evidence cited in support of the theory, and reviewed with commentary in Clark (2013a), includes many neuroimaging experiments showing that activity in lower brain regions reduces as predictions from higher regions improve. These findings have often been interpreted as evidence that feedforward activity signals prediction error. Processes that modulate the precision, reliability, or salience of the feedforward signals are crucial to the theory (Friston, 2010; Brown et al., 2013). Major roles hypothesized for modulation include: context-sensitive sculpting of temporary patterns of effective connectivity within a given network architecture; regulation of the relative strengths of top-down predictions and feedforward data; and off-line use of generative models for both planning actions and understanding the actions of others (Clark, 2013a,b).

The explanatory potential of the theory is therefore wide, and its relevance to core cognitive processes such as attention is discussed in Section 4.5. Nevertheless, although predictive coding theory has been highly influential, there are alternative accounts of some of the main empirical findings that have been cited in its support. In particular, several papers suggest alternative accounts of the reduction in activity as predictability increases. Here we cite only three. Dumoulin and Hess (2006) used functional neuroimaging to study the amount of activity generated in visual cortex when subjects viewed large displays of Gabor line elements oriented either at random or so as to form circular structures. The results confirmed previous findings in showing that activity in visual cortex reduces as statistical structure increases, but they also indicated that this decrease reflects changes in low-level image statistics. They were less consistent with the hypothesis that it reflects feedback from higher levels of scene perception. It was therefore concluded that the contextual effects observed could have been produced by modulatory processes of facilitation and suppression that dpend just upon image statistics and that could operate within V1. There is plenty of other evidence for modulatory interactions within V1. For example, in addition to that already cited in Section 3, Kinoshita, Gilbert, and Das (2009) used intrinsic optical imaging in awake fixating monkeys to measure the strength and cortical distribution of V1 responses to the display of collinear bars. The results confirmed their earlier extracellular electrode recordings in showing that much contextual modulation occurs within V1. Finally, Kok, Jehee, and de Lange (2012) report functional neuroimaging findings suggesting that expectation and task-relevance reduce activity in visual cortex by 'sharpening' it, i.e. by enhancing neural responses that are consistent with expectations or are relevant to the task and suppressing those that are inconsistent or irrelevant. All these findings suggest that standard predictive coding theories may need improvement. One possible improvement is to note that the standard version is simply one way of implementing the computations that predictive coding requires (Spratling, 2013). Furthermore, some of those other versions offer good accounts of much empirical data, including the evidence for intra-regional modulation (e.g. Spratling, 2010). This issue is far from being settled, however, so it is important to note that we have throughout emphasized both feedback and intra-regional interactions, as shown in Figure 1, and have also emphasized evidence for both facilitatory and suppressive forms of modulation. Furthermore, in Section 6.2, we outline a statistical conception of contextual modulation that emphasizes its role in sharpening representations.

One other issue that has been raised in relation to the theory of PEM is also of particular relevance here. It has been argued that PEM cannot explain at least one fundamental feature of human cognition, i.e. the tendency to seek or create certain kinds of complexity, above and beyond whatever is necessary for survival and reproduction (e.g. Little and Sommer, 2013; Phillips, 2013). There are various responses to this 'dark-room problem' (Friston, Thornton, and Clark, 2012). Underlying many of them is an emphasis upon progressive developmental goals of life in addition to the conservative survival goals towards which PEM is primarily directed. The following section therefore considers another information-theoretic perspective on long-term objectives within which 
contextual modulation plays a central role. It is similar to PEM but puts more emphasis upon the open-ended increase of prediction success.

\subsection{Flourishing by increasing prediction success}

Neurocomputational studies of the long-term objective of coherent information maximization, referred to as coherent infomax, have shown formally how contextual modulation can be used to guide both learning and on-going processing (Phillips, Kay and Smyth, 1995; Kay, Floreano and Phillips, 1998). This information-theoretic objective was explicitly formulated for local neural processors that receive a distinct class of modulatory inputs in addition to the RF inputs that specify their selectivity. Learning rules for modifying the strengths of their synaptic connections were derived analytically from the formally-specified objective, and shown to have biological plausibility. It was shown that contextual modulation was required to achieve the objective (Smyth, Phillips and Kay, 1996), and that the learning rules can be applied exactly in simple cases (Kay, Floreano and Phillips, 1998) and by approximation in more complex cases (Kay and Phillips, 2011). The dynamics of systems built from such local neural processing elements were shown to depend in functionally useful ways upon predictive relationships between the variables defined on their driving inputs. They can discover variables that predict pre-specified variables, such as primary reinforcers. This is equivalent to supervised learning. Exactly the same learning rules can also discover new variables simply by discovering the predictive relations between them. In statistical terms this is a form of non-linear latent structure analysis. It is equivalent to unsupervised learning. The knowledge thus acquired is embodied in the strengths of the synaptic connections mediating RF and contextual inputs. Neural network simulations show that it sharpens representations by amplifying coherent activities and suppressing incoherent activities (Kay, Floreano and Phillips, 1998). The many recent empirical findings reviewed in Sections 3, 4, and 5 clearly support and develop this conception of contextual modulation. Contextual modulation has therefore been rigorously related to the long-term objective of maximizing coherent information transmission, and thus of maximizing prediction success.

It could be argued that information-theoretic concepts, such as Shannon entropy, are of limited use in psychology and neurobiology because they quantify information in a way that depends only on the probability of events and ignores their 'meaning'. If predictive relationships are central to meaning, however, then our emphasis upon coherent information transmission may help to overcome that limitation.

One major problem faced by previous versions of the theory of coherent information maximization concerns the hypothesis that NMDA receptor channels provide the mechanism for the contextual modulation on which the theory relies. The problem is that the theory requires the modulatory and driving inputs to come from different sources and to be integrated separately prior to their interaction (Kay and Phillips, 2011). NMDARs are co-localized with AMPA receptors, however, so they cannot receive inputs from sources that differ from AMPA receptors, and they cannot first be integrated separately before being used to modulate response to the integrated RF input. We now see that, as suggested by a theory of learning closely related to coherent infomax (Körding and König, 2000), a solution to this problem is provided by the evidence for apical amplification (Larkum, 2013; Palmer et al, 2014). The RF inputs from the basal dendrites are integrated at the somatic spike initiation zone. The contextual inputs from the distal tuft dendrites are integrated at the calcium spike initiation zone, and this then modulates the cell's response through calcium spikes transmitted to the soma by the apical dendrite. A conductance based model of what we call apical amplification, when implemented via the BAC mechanism, has shown that it can simulate many of the modulatory effects described above (Siegel, Körding, and König, 2000). It shows that the operation of this mechanism has greater effects on burst probability than on spike rates, and that it enhances inter-areal coupling. Thus, apical amplification could enable the integrated contextual inputs to modulate response to the integrated RF inputs as the theory of coherent infomax 
requires. As the theoretical studies of coherent infomax and the empirical studies of BAC firing were carried out independently the apparent closeness of their match encourages the hope that they reflect a common distal reality. That conclusion cannot be drawn with confidence yet, however, because the technical difficulties of studying dendritic processes are so great, particularly in awake behaving states, that much remains to be discovered concerning the exact division of labour between somatic and apical spike initiation zones. In the meantime, our working assumption is that conceptions of contextual modulation will lead to a better understanding of the functional significance of mechanisms for apical amplification, such as BAC firing.

There are many similarities between the theories of PEM and coherent information transmission (Kay and Phillips, 2011; Phillips, 2013). Both theories propose a single information-theoretic objective towards which the structures and dynamics of neural systems are directed. Both assume driving and modulatory inputs to neural processors; both describe the system dynamics as moving towards states in which their predictions are satisfied. In both theories the selective transmission of feedforward information depends on contextual modulation within and between regions. Indeed, if the amount of information in the predictions is assumed to be constant, then the system level goal of free energy reduction can be shown to formally imply coherent information transmission at the level of local neural processors. This is because prediction success is then the complement of prediction error (Friston, personal communication). That assumption may not always be valid, however, so the two theories are not in general equivalent, and there are some important differences. The difference of most importance here concerns their objectives. Free energy theory emphasizes reducing deviations from homeostasis by reducing prediction error. That seems to imply an optimum state in which there are no errors. Errors may be reduced by keeping the predictions as simple as survival and reproduction allow, as much discussed in relation to 'the dark room problem' (Friston, Thornton and Clark, 2012; Clark, 2013a; Little and Sommer, 2013). In contrast to that, the goal of increasing coherent information transmission is more open-ended. It implies that the amount of information correctly predicted could go on increasing indefinitely, so agents with that objective should both seek and create organized complexity. Thus, prima facie, emphasis upon the reduction of prediction error seems better suited to expressing conservative homeostatic goals, whereas emphasis upon increasing prediction success seems better suited to expressing progressive goals that increase the richness and accuracy of the predictions made. Things may not be that simple, however, and such issues are discussed in greater depth in Clark (2013a, b).

\subsection{Increasing harmony and reducing conflict in mental life}

The generic computations that are involved in predictive processing can be related to subjective aspects of individual experience, such as self-identity, body ownership, and the sense of agency. This can be done via a theory of interoceptive inference (Seth, Suzuki and Critchley, 2011, Seth, 2013). As in visual perception, interoceptive inference is hypothesized to involve a hierarchy of topdown predictions that guide the interpretation of bottom-up signals. The subjective sense of the reality of the self and of the external world is hypothesized to depend on the successful suppression of interoceptive signals by precise top-down predictions (Seth, Suzuki and Critchley, 2011). Similarly, the subjective sense of agency is hypothesized to arise from precise predictions of the sensory consequences of actions (Fletcher and Frith, 2009). The theory of interoceptive inference synthesizes much evidence from phenomenology, neurobiology, and psychopathology. It argues that the experience of body ownership depends upon the integration of precision-weighted interoceptive and exteroceptive signals (Seth, 2013). These signals are transmitted to higher cortical regions by Layer 2/3 pyramidal cells whose post-synaptic gain is modulated by contextual variables such as those emphasized above. Furthermore, this perspective also connects deficits in interoceptive inference with specific psychiatric disorders, and schizophrenia in particular (Seth, Suzuki and Critchley, 2011). Seth et al emphasize the role of the classic neuromodulators, but locally-specific intracortical contextual modulation must also be involved. Furthermore, the driving activities that 
must be coordinated can have internal as well as external origins. Thus, in addition to harmonizing interoceptive predictions with interoceptive signals, contextual modulation is required to harmonize thoughts with each other, as well as with emotions and actions (Martin and Pacherie, 2013).

\section{Assessment of our hypotheses and their prospects}

There are many ways in which the hypotheses outlined here require further testing and development. For example, we have presented evidence for both local circuit and intracellular mechanisms, but we know of no empirical or theoretical studies of how they are combined. That is clearly a major issue that needs to be resolved. Other problems and prospects are as follows.

\subsection{Are drive and modulation clearly distinct?}

A possible weakness of coherent information theory and our neuron-centric conception of contextual modulation is that they imply a dichotomy between driving and contextual inputs. Can that dichotomy be sustained? Can contextual modulation be clearly distinguished from dynamic changes of RF selectivity? Some authorities prefer to include contextual inputs within a broad conception of the receptive field (e.g. Gilbert, 2013). In doing so they preserve the basic distinction between modulatory and classical components of the RF input, however, so that is simply a matter of terminology. An important source of evidence on the assumption of a dichotomy between RF and contextual inputs comes from studies of intracellular mechanisms for apical amplification, such as BAC firing. They suggest that inputs to dendrites on the apical tuft affect the cell's output in a way that is clearly functionally distinct from that of the basal synapses that are electrically closer to the somatic spike initiation zone (Larkum, 2013).

It is possible in principle to combine drive and modulation to different degrees in different synaptic connections, but if that were so their distinct contributions could still be distinguished (Smyth, Phillips, and Kay, 1996). Where this possibility has been studied empirically, however, e.g. in mouse visual (De Pasquale and Sherman, 2011) and auditory (Covic et al., 2011) cortex, all the synaptic connections were categorically either driving or modulatory. Whether there are also cortical synapses in which drive and modulation are combined remains to be seen. Even if there are it would still be possible for drive and modulatory interactions to be categorically distinguished by mechanisms such as that of apical amplification.

\subsection{How does contextual modulation operate within heterarchical architectures?}

Section 6.1 noted that the control of precision, which is closely related to contextual modulation, is central to prediction-error minimization in the theory of free energy reduction. That theory assumes hierarchical architectures, however, and there is evidence that human large-scale functional networks form small-world heterarchical architectures, rather than simple hierarchies (Bressler and Menon, 2010). Furthermore, computational models show that simple hierarchies do not provide a good fit to response latencies in vision (Capalbo et al., 2008). Theories emphasizing prediction-error minimization therefore need to be modified so that they can apply to small-world heterarchies. This problem seems less relevant to the use of contextual modulation to maximize coherent information transmission, as discussed in Section 6.2. That does not assume hierarchies, but we know of no computational studies of its use in heterarchical small-world architectures.

\subsection{Are there major transitions in the evolution of inferential and modulatory capabilities?}

Cognitive capabilities change over the course of evolution and over the course of an individual's life-span. Such changes typically include both conservation and progression. Our working assumption is therefore that more recently evolved and more mature neural systems preserve simpler forms of contextual modulation, while adding more demanding but more capable forms. Possible evolutionary transitions in these capabilities have been proposed on the grounds of probability theory (Phillips 2012). Those proposals were speculative but studies of synaptic evolution are already 
providing encouraging findings. For example, Nithianantharajah et al. (2013) studied the effects of mutations in genes coding for post-synaptic proteins that regulate NMDAR function on the behavior of mice. They adapted the CANTAB tests of conditioning, complex learning, attention, and cognitive control for use with mice. Their findings showed that the mutations affected those functions in much the same way in mice as they do when inherited by humans. This suggests that the cognitive functions of NMDAR regulatory proteins emerged sometime between 550 and 90 million years ago. Studies of extensive evolutionary divergences in NMDAR subtypes that have occurred since then strengthen and develop these conclusions (e.g. Ryan et al., 2013). Furthermore, evolutionary developments involving expression of genes affecting NMDAR function have occurred specifically in the human lineage within the last six million years (Konopka et al., 2012). The cognitive functions affected by these mutations are likely to involve contextual modulation, and this inference is supported by their strong association with schizophrenia in humans (Nithianantharajah et al., 2013). None of the cognitive tests used in those studies allow rigorous conclusions concerning the generic forms of contextual modulation outlined in Section 3, however. So what we now need is a battery of psychophysical tests of those capabilities for use in a wide range of species. The evolutionary increase in the molecular complexity of modulatory post-synaptic mechanisms and their regulation suggests that if such test batteries were combined with genetic manipulations and cross-species comparisons a far richer account of contextual modulation would be revealed than that hypothesized here.

We know of no direct studies of the evolutionary development of apical amplification and its role in contextual modulation. The distribution and variation of pyramidal cell morphology and function across species provides some clues, however. Pyramidal neurons are abundant in mammals, birds, fish and reptiles, but not in amphibians (Spruston, 2008). In mammals they are found in forebrain structures, such as the cerebral cortex, hippocampus and amygdala, but not in the olfactory bulb, striatum, midbrain, hindbrain or spinal cord (Spruston, 2008). Thus, they are found primarily in structures that are associated with advanced cognitive functions. This suggests that their core functions have been preserved, while they have also evolved to enhance inferential capabilities in ways that remain to be elucidated.

\subsection{Scaffolded Minds: Can higher forms of human flourishing be characterized by information-theoretic objectives?}

Long-term objectives that may be served by contextual modulation were hypothesized in Section 6, but can any such objective be immune to 'information-theoretic subversion'? Consider a system aiming to maximize prediction success. It may well exhibit some kind of ongoing expansion by continually extending its reach to encompass new states of organized complexity. But there will be many ways to do this. Such an agent, for example, might play a computer game continuously rearranging a finite (but lifetime inexhaustible) stock of small coloured tiles into larger and larger patterns, inducing then learning new predictive relationships at every stage. The creation and transmission of coherent relationships between informative signals within and between the agent's neural economy and its environment may then increase at every step, but its quality of life may still fall far short of 'higher' forms of human flourishing.

More may therefore be required of objectives that claim to characterize such aspects of human flourishing. Clues to identifying them may lie in the complex structured social practices and physical environments, that we humans create, transmit, and progressively alter. Humans induce complex multi-layered world models to enhance adaptive success, but as a spin-off, we became able to build complex social and physical environments. When marinated in the unique statistical baths of such designer worlds, minds like ours spawn new local goals and projects. We build worlds that enable us to piggy-back the satisfaction of our basic needs upon the exploration of complex rewarding spaces the spaces of art, science, and culture. By designing, re-designing, and re-re-designing our own environments we continually move the goalposts for our own prediction-based learning. Our 
evolving cultural practices thus generate minds that go where no human minds have gone before. In doing so they acquire newly specialized capabilities and ways of coordinating them using contextual modulation. Most of the heavy lifting, when it comes to explaining the shape and nature of our modern minds, however, is done by our individual social and intellectual histories - histories that are replete with chance and path-dependent unfolding (Clark, 2013a). Therefore, from the viewpoint of individuals, and the societies that they create, context-sensitive probabilistic inference may be important, not because it serves the formal long-term objectives hypothesized above, but because it enables them create, modify, and pursue their own endlessly variable goals.

\section{ACKNOWLEDGEMENTS}

We thank Michael Wibral for Figure 2 and for valuable discussions. We also thank Andre Bastos, Will Barnes, Ernest Greene, Jim Kay, Matthew Larkum, Bartlett Mel, Lars Muckli, Heiko Neumann, Lucy Petro, Viola Priesmann, Wolf Singer, and Mike Spratling for comments on issues discussed in earlier versions of this paper. Thanks are due also to the editor Francesca Cirulli, and to three anonymous reviewers who provided valuable comments on an earlier version of the paper.

\section{REFERENCES}

Adams, R. A., Stephan, K. E., Brown, H. R., Frith, C. D., \& Friston, K. J. (2013). The computational anatomy of psychosis. Front. Psychiatry, 4. doi: 10.3389/fpsyt.2013.00047

Adesnik, H., Bruns, W., Taniguchi, H., Huang, J., \& Scanziani, M. (2012). A neural circuit for spatial summation in visual cortex. Nature, 490, 226-231.

Allman, J., Miezin, F., \& McGuinness, E. (1985). Stimulus specific responses from beyond the classical receptive field: neurophysiological mechanisms for local-global comparisons in visual neurons. Ann. Rev. Neurosci., 8, 407-430.

Andreou, C., Tsapkini, K., Bozikas, V. P., Giannakou, M., Karavatos, A., \& Nimatoudis, I. (2009). Effects of sentence context on lexical ambiguity resolution in patients with schizophrenia. Neuropsychologia, 47, 1079-1087. doi:10.1016/j.neuropsychologia.2008.12.031.

Angelucci, A., Levitt, J.B, Walton, E.J, Hupe, J. M, Bullier, J., Lund J.S. (2002). Circuits for local and global signal integration in primary visual cortex. J. Neurosci. 22, 8633-8646.

Atallah, B. V., Bruns, W., Carandini, M., \& Scanziani, M. (2012). Parvalbumin-expressing interneurons linearly transform cortical responses to visual stimuli. Neuron, 73, 159-170.

Ayaz, A., Chance, F. S. (2009) Gain modulation of neuronal responses by subtractive and divisive mechanisms of inhibition. J. Neurophysiol. 101, 958-968. doi:10.1152/jn.90547.2008.

Bachmann, T., \& Hudetz, A. G. (2014). It is time to combine the two main traditions in the research on the neural correlates of consciousness: C= L× D. Front. Psychol., 5. doi:10.3389/fpsyg.2014.00940.

Bar, M. (2004). Visual objects in context. Nat. Rev. Neurosci., 5, 617-629.

Bastos, A. M., Vezoli, J., Bosman, C. A., Schoffelen, J-M., Oostenveld, R., Dowdall, J. R., De Weerd, P., Kennedy, H. \& Fries, P. (2015). Visual Areas Exert Feedforward and Feedback Influences through Distinct Frequency Channels, Neuron 85, 1-12. http://dx.doi.org/10.1016/j.neuron.2014.12.018

Bauer, R., \& Heinze, S. (2002). Contour integration in striate cortex: Classic cell responses or cooperative selection? Experimental Brain Research, 147, 145-152.

Bayerl, P., \& Neumann, H. (2004). Disambiguating visual motion through contextual feedback modulation. Neural Comp., 16, 2041-66.

Bayerl, P., \& Neumann, H. (2007). A fast biologically inspired algorithm for recurrent motion estimation. IEEE Transactions on Pattern Analysis and Machine Intelligence, 29, 246260. 
Behrens, M. M., \& Sejnowski, T. J. (2009). Does schizophrenia arise from oxidative dysregulation of parvalbumin-interneurons in the developing cortex? Neuropharmacology, 57, 193-200.

Behabadi, B. F., A. Polsky, A., Jadi, M., J. Schiller, J., \& Mel, B. W. (2012). Location-dependent excitatory synaptic interactions in pyramidal neuron dendrites. PLoS Comput. Biol., vol. 8, no. 7, 2012, e1002599.

Bellman, R. E. (1961). Adaptive Control Processes. Princeton, NJ: Princeton Univ. Press,

Bisley, J.W., \& Goldberg, M.E. (2006). Neural correlates of attention and distractibility in the lateral intraparietal area. J. Neurophysiol., 95, 1696-1717.

Boudewijns, Z. S. R. M., Groen, R. M., Lodder, B., Minni T. B. McMaster, M. T. B., Kalogreades, L., de Haan, R., Narayanan, R. T., Meredith, R. M., Huibert D. Mansvelder, H. D. \& de Kock, C. P. J. (2013). Layer-specific high-frequency action potential spiking in the prefrontal cortex of awake rats. Front. Cell. Neurosci. 7, 99. doi: 10.3389/fncel.2013.00099

Braitenberg, V., \& Schuz, A. (1991). Anatomy of the Cortex. Berlin: Springer-Verlag.

Braff, D. L., Geyer, M. A., \& Swerdlow, N. R. (2001). Human studies of prepulse inhibition of startle: normal subjects, patient groups, and pharmacological studies. Psychopharmacology (Berl), 156, 234-258.

Bressler, S. L., \& Menon, V. (2010). Large-scale brain networks in cognition: emerging methods and principles. Trends Cogn. Sci., 14, 277-290.

Brown, H., Adams, R. A., Parees, I., Edwards, M., and Friston, K. (2013). Active inference, sensory attenuation and illusions. Cogn. Process. 14, 411-427. DOI 10.1007/s10339-013-0571-3

Butler, P. D., Abeles, I. Y., Silverstein, S. M., Dias, E. C., Weiskopf, N. G., Calderone, D. J., \& Sehatpour, P. (2013). An event-related potential examination of contour integration deficits in schizophrenia. Front. Psychol., 4:132. doi: 10.3389/fpsyg.2013.00132.

Buzsáki, G. (2006). Rhythms of the brain. New York: Oxford University Press.

Capalbo, M., Postma, E., Goebel, R. (2008). Combining structural connectivity and response latencies to model the structure of the visual system. PLoS Comput. Biol., 4, e1000159

Carandini, M., \& Heeger, D.J. (2012). Normalization as a canonical neural computation. Nat. Rev. Neurosci., 13, 51-62.

Cardin, J.A., Carle’ N, M., Meletis, K., Knoblich, U., Zhang, F., Deisseroth, K., Tsai, L.-H., \& Moore, C.I. (2009). Driving fast-spiking cells induces gamma rhythm and controls sensory responses. Nature, 459, 663-667.

Castellano, M., Plöchl, M., Vicente, R., \& Pipa, G. (2014). Neuronal oscillations form parietal/frontal networks during contour integration. Front. Integ. Neurosci, 8. doi: 10.3389/fnint.2014.00064

Chawla, D., Lumer, E. D., \& Friston, K. J. (1999). The relationship between synchronization among neuronal populations and their mean activity levels. Neural Computation, 11, 1389-1411.

Chen, C. C., \& Tyler, C. W. (2008). Excitatory and inhibitory interaction fields of flankers revealed by contrast-masking functions. J. Vis., 8(4):10, 1-14, http://journalofvision.org/8/4/10/, doi:10.1167/8.4.10.

Chen, Y., Norton, D., \& Ongur, D. (2008). Altered center-surround motion inhibition in schizophrenia. Biol Psychiatry., 64, 74-77. doi: 10.1016/j.biopsych.2007.11.017

Cisek, P., \& Kalaska, J. F. (2010). Neural mechanisms for interacting with a world full of action choices. Annu. Rev. Neurosci. 33, 269-298.

Clark, A. (2001). Mindware: An introduction to the philosophy of cognitive science. New York: Oxford University Press.

Clark, A. (2013a). Whatever Next? Predictive Brains, Situated Agents, and the Future of Cognitive Science. Behav. Brain Sci.. 36, 131-204.

Clark, A. (2013b) The Many Faces of Precision. Front. Psychology 4:270. doi:10.3389/fpsyg.2013.00270

Clowes, M. B. (1971). On seeing things. Art Intell.79-116. 
Cobb, S. R., Buhl, E. H., Halasy, K., Paulsen, O., \& Somogyi, P. (1995). Synchronization of neuronal activity in hippocampus by individual GABAergic interneurons. Nature, 378, 75-78.

Covic, E. N. \& Sherman, S. M. (2011). Synaptic properties of connections between the primary and secondary auditory cortices in mice. Cerebral Cortex, 21, 2425-2441. doi:10.1093/cercor/bhr029

Cuntz, H., Forstner, F., Borst, A., Häusser, M. (2010) One Rule to Grow Them All: A General Theory of Neuronal Branching and Its Practical Application. PLoS Comput Biol, 6: e1000877. doi: 10.1371/journal.pcbi.1000877

Dakin, S., Carlin, P., \& Hemsley, D. (2005). Weak suppression of visual context in chronic schizophrenia. Curr. Biol., 15:R822-R824.

Davenport, J. L., \& Potter, M. C. (2004). Scene consistency in object and background perception. Psychol. Sci., 15, 559-564.

Das, A., \& Gilbert, C. D. (1995). Long-range horizontal connections and their role in cortical reorganization revealed by optical recording of cat primary visual cortex. Nature, 375,780-784.

Dayan, P. (2012). Twenty-five lessons from computational neuromodulation. Neuron, 76, 240-256.

De Meyer, K. and Spratling, M. W. (2009). A model of non-linear interactions between cortical topdown and horizontal connections explains the attentional gating of collinear facilitation. Vision Res., 49, 553-68.

De Pasquale, R. \& Sherman, S. M. (2011). Synaptic properties of corticocortical connections between the primary and secondary visual cortical areas in the mouse. J. Neurosci., 31, $16494-$ 16506.

Desimone, R. \& Duncan, J. (1995). Neural mechanisms of selective visual attention. Ann. Rev. Neurosci., 18, 193-222.

DiCarlo, J.J., Zoccolan, D., \& Rust, N.C. (2012). How does the brain solve visual object recognition? Neuron, 73, 415-434.

Doniger, G. M., Foxe, J. J., Murray, M. M., Higgins, B. A., \& Javitt, D. C. (2002). Impaired visual object recognition and dorsal/ventral stream interaction in schizophrenia. Arch. Gen. Psychiatry, 59, 1011-1120

Douglas, R. J., \& Martin, K. A. C. (2007). Mapping the matrix: The ways of neocortex. Neuron, 56, 226-238.

Douglas, R. J., \& Martin, K. A. C. (2008). Recurrent neuronal circuits in the neocortex. Curr. Biol., 17 No 13, R496.

Dumoulin, S. O., \& Hess, R. F. (2006). Modulation of V1 activity by shape: image-statistics or shape-based perception? J. Neurophysiol., 95, 3654 -3664. doi:10.1152/jn.01156.2005.

Egner, T., and Hirsch, J. (2005). Cognitive control mechanisms resolve conflict through cortical amplification of task-relevant information. Nat. Neurosci., 8, $1784-1790$.

Engel, A., \& Singer, W. (2001). Temporal binding and the neural correlates of sensory awareness. Trends Cogn. Sci., 5, $16-25$.

Feldman, H., and Friston, K. J. (2010). Attention, uncertainty, and free-energy. Front. Human Neurosci. , 4, 215, doi: 10.3389/fnhum.2010.00215

Fiorani, M., Rosa, M. G., Gattass, R., \& Rocha-Miranda, C. E. (1992). Dynamic surrounds of receptive fields in primate striate cortex: a physiological basis for perceptual completion? Proc. Natl. Acad. Sci. USA, 89, 8547-8551.

Fiorillo, C. D. (2012). On the need for a unified and Jaynesian definition of probability and information within neuroscience. Information, 3:175-203.

Fitzpatrick, D. (1996). The functional organization of local circuits in visual cortex: insights from the study of tree shrew striate cortex. Cereb Cortex, 6, 329-341.

Fize, D., Cauchoix, M., \& Fabre-Thorpe, M. (2011). Humans and monkeys share visual representa tions. Proc. Natl. Acad. Sci. U.S.A., 108, 7635-7640.doi:10.1073/pnas. 1016213108. 
Fletcher, P. C., \& Frith, C. D. (2009). Perceiving is believing: a Bayesian approach to explaining the positive symptoms of schizophrenia. Nat. Rev.Neurosci., 10, 48-58.

Flevaris, A. V., Martínez, A., \& Hillyard, S. A. (2013). Neural substrates of perceptual integration during bistable object perception. J. Vis., 13, 17, 1-25.

Fries, P., Neuenschwander, S., Engel, A. K., Goebel, R., and Singer, W. (2001a). Rapid feature selective neuronal synchronization through correlated latency shifting. Nat. Neurosci., 4, 194200.

Fries, P., Nikolic, D., Singer, W. (2007). The gamma cycle. Trends Neurosci., 30, 309-316.

Fries, P, Reynolds, J. H., Rorie, A. E., and Desimone, R. (2001b). Modulation of oscillatory neuronal synchronization by selective visual attention. Science, 29, 1560-1563.

Friston, K. (2009) The free-energy principle: A rough guide to the brain? Trends Cog. Sci., 13, 293301.

Friston, K. J. (2010). The free-energy principle: a unified brain theory? Nat. Rev. Neurosci., 11, 127138.

Friston, K. J. (2013). Life as we know it. J R Soc Interface 10: 20130475. http://dx.doi.org/10.1098/rsif.2013.0475

Friston, K. J., \& Stephan, K. E. (2007). Free energy and the brain. Synthese, 159, 417-458. doi:10.1007/s11229-007-9237-y

Friston K. J., Thornton C., Clark A. (2012). Free-energy minimization and the dark-room problem. Front. Psychol., 3, 130.10.3389/fpsyg.2012.00130

Gail, A., Brinksmeyer, H. J., \& Eckhorn, R. (2000). Contour decouples contour activity across texture representation in monkey striate cortex. Cerebral Cortex, 10, 840-850.

Gilbert, C. D. (2013). Contextual modulation in the visual cortex. J. Vis., 13, 1369, doi: 10.1167/13.9.1369

Gilbert, C. D. \& Sigman, M. (2007). Brain States: Top-Down Influences in Sensory Processing. Neuron, 54, 677-696.

Godfrey-Smith, P. (1996). Complexity and the Function of Mind in Nature. Cambridge: Cambridge University Press.

Gonzalez-Burgos, G., \& Lewis, D. A. (2012). NMDA receptor hypofunction, parvalbumin-positive neurons, and cortical gamma oscillations in schizophrenia. Schizophr. Bull., 38, 950-957. doi: $10.1093 / \mathrm{schbul} / \mathrm{sbs} 010$

Graham, K. T., Martin-Iverson, M. T., Holmes, N. P., Jablensky, A., \& Waters, F. (2014). Deficits in agency in schizophrenia, and additional deficits in body image, body schema, and internal timing, in passivity symptoms. Front Psychiatry, 5, 126. doi: 10.3389/fpsyt.2014.00126.

Gregoriou, G. G., Gotts, S. J., Zhou, H., \& Desimone, R. (2009). High-frequency, long-range coupling between prefrontal and visual cortex during attention. Science, 324,1207-1210.

Haider, B. \& McCormick, D. A. (2009). Rapid neocortical dynamics: Cellular and network mechanisms. Neuron, 62, 171-189.

Hancock, P. J., \& Phillips, W. A. (2004). Pop-out from abrupt visual onsets. Vis. Res., 44, 22852299.

Hancock, P. J. B., Walton, L., Mitchell, G., Plenderleith, Y., \& Phillips, W. A. (2008). Segregation by onset asynchrony. J. Vis., 8, 21, 1-21, doi:10.1167/8.7.21.

Heeger, D. J. (1992). Normalization of cell responses in cat striate cortex. Vis. Neurosci., 9, 181197.

Huang, P. C., Hess, R. F., \& Dakin, S. C. (2006). Flank facilitation and contour integration: Different sites. Vis. Res., 46, 3699-3706.

Hughes, E. G., Peng, X., Gleichman, A. J., Lai, M., Zhou, L., Tsou,R., Parsons,T. D., Lynch, D. R., Dalmau, J. \& Balice-Gordon, R.J. (2010). Cellular and synaptic mechanisms of anti-NMDAR encephalitis. J. Neurosci., 30, $5866-5875$. 
Ito, M, \& Gilbert, C. D. (1999) Attention modulates contextual influences in the primary visual cortex of alert monkeys. Neuron, 22, 593-604.

Jadi, J. P., Behabadi, B. F., Poleg-Polsky, A., Schiller, J., and Mel, B. W. (2014). An augmented two-layer model captures nonlinear analog spatial integration effects in pyramidal neuron dendrites. Proc. IEEE, 102, 782-798.

Jentsch, J. D., \& Roth, R. H. (1999). The neuropsychopharmacology of phencyclidine: from NMDA receptor hypofunction to the dopamine hypothesis of schizophrenia. Neuropsychopharmacology, 20, 201-225.

Kantrowitz, J. T., \& Javitt, D. C. (2010). N-methyl-d-aspartate (NMDA) receptor dysfunction or dysregulation: the final common pathway on the road to schizophrenia? Brain Res. Bull., 83, $108-121$.

Kapadia, M. K., Ito, M., Gilbert, C. D., \&Westheimer, G. (1995). Improvement in visual sensitivity by changes in local context: parallel studies in human observers and in V1 of alert monkeys. Neuron 15, 843-856.

Kay, J., Floreano, D., \& Phillips, W. A. (1998). Contextually guided unsupervised learning using local multivariate binary processors. Neural Networks, 11, 117-140.

Kay, J., \& Phillips, W. A. (2011). Coherent infomax as a computational goal for neural systems. Bull. Math. Biol., 73, 344-372, doi 10.1007/s11583-010-9564-x.

Kéri, S., Kelemen, O., Benedek, G., \& Janka, Z. (2005). Lateral interactions in the visual cortex of patients with schizophrenia and bipolar disorder. Psychol. Med. 35, 1043-1051

Kéri, S., Kelemen, O., \& Benedek, G. (2009). Attentional modulation of perceptual organisation in schizophrenia. Cogn. Neuropsychiatry, 14, 77-86. doi: 10.1080/13546800902757936.

Kim, T., \& Freeman, R. D. (2014). Selective stimulation of neurons in visual cortex enables segregation of slow and fast connections. Neurosci. 274, 170-186. doi: 10.1016/j.neuroscience.2014.05.041

Kinoshita, M., Gilbert, C. D., \& Das, A. (2009) Optical imaging of contextual interactions in V1 of the behaving monkey. J. Neurophysiol., 102, 1930-1944.

Klemm, W. R., Li, T. H., \& Hernandez, J. L. (2000). Coherent EEG indicators of cognitive binding during ambiguous figure tasks. Consciou. Cogn., 9, 66-85.

Klink, P. C., van Wezel, R. J. A. \& van Ee, R. (2012). United we sense, divided we fall: contextdriven perception of ambiguous visual stimuli. Phil. Trans. R. Soc. B, 367, 932-941. (doi:10.1098/rstb.2011.0358)

Koch, C. (2004). The Quest for Consciousness. Englewood, CO: Roberts and Co.

Kok, P., Jehee, J. F., \& de Lange, F. P. (2012). Less is more: expectation sharpens representations in the primary visual cortex. Neuron, 75, 265-270.

Konopka, G., Friedrich, T., Davis-Turak, J., Winden, K., Oldham, M. C., et al. (2012). Humanspecific transcriptional networks in the brain. Neuron, 75, 601-617.

Körding, K. P. \& König, P. (2000) Learning with two sites of synaptic integration. Network: Computation in Neural Systems, 11,1-15.

Kouh, M., \& Poggio, T. (2008). A canonical neural circuit for cortical nonlinear operations. Neural Comput. 20, 1427-1451.

Krüger, N., and Wörgötter, F. (2005). Multi-modal primitives as functionalmodels of hypercolumns and their use for contextual integration. In: Proceedings of the 1st international symposium on brain, vision and artificial intelligence, LNCS 3704. Springer, Berlin HeidelbergNew York, p 157-166.

Lamme, V. A. F. (2004). Beyond the classical receptive field: Contextual modulation of V1 responses. In J. S. Werner, \& L. M. Chalupa (Eds.) The Visual Neurosciences (pp. 720-732). Cambridge, MA: MIT Press.

Lamme, V. A. F. \& Spekreijse, H. (1998) Neuronal synchrony does not represent texture segregation. Nature, 396, 362-366. 
Landauer, T. K. \& Dumais, S. T. (1997). A solution to Plato's problem: The Latent Semantic Analysis theory of acquisition, induction, and representation of knowledge. Psych. Rev., 104, 211-240.

Larkum, M. (2013). A cellular mechanism for cortical associations: an organizing principle for the cerebral cortex. Trends Neurosci., 36,141-151.

Larkum, M. E. Nevian, T., Sandler, M., Polsky, A., and Schiller J. (2009) Synaptic integration in tuft dendrites of layer 5 pyramidal neurons: A new unifying principle. Science, 325, 756-760.

Larkum, M. E. Senn, W., \& Lüscher, H-R. (2004). Top-down dendritic input increases the gain of layer 5 pyramidal neurons. Cerebral Cortex, 14:1059-1070; doi:10.1093/cercor/bhh065

Larkum, M. E., Zhu, J. J., and Sakmann, B. (1999). A new cellular mechanism for coupling inputs arriving at different cortical layers. Nature, 98, 6725, 338-341.

Lawson, R. P., Rees, G., \& Friston, K. J. (2014). An aberrant precision account of autism. Frontiers in human neuroscience, 8. doi: 10.3389/fnhum.2014.00302

Lee, C. C. \& Sherman, S. M. (2010) Drivers and modulators in the central auditory pathways. Front. Neurosci., 4, 1: 79-86. doi: 10.3389/neuro.01.014.2010

Lee, H., Dvorak, D., Kao, H. Y., Duffy, A. M., Scharfman, H. E., and Fenton, A. A. (2012a): Early cognitive experience prevents adult deficits in a neurodevelopmental schizophrenia model. Neuron, 75, 714-724.

Lee, S.-H., Kwan, A. C., Zhang, S., Phoumthipphavong, V., Flannery, J. G., Masmanidis, S. C., Taniguchi, H., Huang, Z. J., Zhang, F., Boyden, E. S., Deisseroth, K., \& Dan, Y. (2012b). Activation of specific interneurons improves V1 feature selectivity and visual perception. Nature, 488, 379-383.

Lesh, T. A., Niendam, T. A., Minzenberg, M. J., \& Carter, C. S. (2011). Cognitive control deficits in schizophrenia: mechanisms and meaning. Neuropsychopharmacology, 36, 316-338.

Lewis, D. A., Curley, A. A., Glausier, J. R., \& Volk, D. W. (2012). Cortical parvalbumin interneurons and cognitive dysfunction in schizophrenia. Trends Neurosci., 35, 57-67. doi: 10.1016/j.tins.2011.10.004.

Lima, B., Singer, W., Chen, N. H., Neuenschwander, S. (2010). Synchronization dynamics in response to plaid stimuli in monkey V1. Cereb. Cortex, 20, 1556-1573.

Lindenmayer, J. P., Bernstein-Hyman, R., \& Grochowski, S. (1994). A new five factor model of schizophrenia. Psychiatr. Q., 65, 299-322.

Little, D. Y-J., \& Sommer, F. T. (2013). Maximal mutual information, not minimal entropy, for escaping the "Dark Room". Behav. Brain Sci. 36, 220-221.

Liu, H., \& Singh, P. (2004). ConceptNet: A practical commonsense reasoning toolkit,” BT Technology J., 22, 211-226.

Lund, J. S., Angelucci, A., \& Bressloff, P. C. (2003) Anatomical Substrates for Functional Columns in Macaque Monkey Primary Visual Cortex. Cerebral Cortex, 12, 15-24.

Maehara, G., Huang, P-C., \& Hess R. F. (2010). The effects of flankers on contrast detection and discrimination in binocular, monocular, and dichoptic presentations. J. Vis., 10, 13, 1-15.

Major, G., Larkum, M. E., and Schiller, J. (2013). Active properties of neocortical pyramidal neuron dendrites. Annu. Rev. Neurosci., 36, 1-24.

Mandon, S., and Kreiter, A. K. (2005). Rapid contour integration in macaque monkeys. Vis. Res. 45, 291-300.

Marcus, D. S., \& Van Essen, D. C. (2002). Scene segmentation and attention in primate cortical areas V1 and V2. J. Neurophys., 88, 2648-2658.

Mareschal, I., \& Clifford, C. W. G. (2013). Spatial structure of contextual modulation. J. Vis. 13, 2, $1-9$.

Markov, N. T. \& Kennedy, H. (2013) The importance of being hierarchical. Curr. Opin. Neurobiol., 23, 187-194.

Marr, D. (1982). Vision. San Fancisco, CA: W. H. Freeman and Co. 
Martel G., Dutar P., Epelbaum J., Viollet C. (2012). Somatostatinergic systems: an update on brain functions in normal and pathological aging. Front. Endocrinol., 3, 154. doi:10.3389/fendo.2012.00154

Martin, J-R., and Pacherie, E. (2013). Out of nowhere: Thought insertion, ownership and contextintegration. Conscious. Cogn., 22, 111-122.

Massoudi, R., Van Wanrooij, M. M., Van Wetter, S. M. C. I., Versnel, A., \& Van Opstal, A. J. (2013). Stable bottom-up processing during dynamic top-down modulations in monkey auditory cortex. European J. Neurosci., 1-13. doi:10.1111/ejn.12180

Melloni, L., Molina, C., Pena, M., Torres, D., Singer, W., \& Rodriguez, E. (2007). Synchronization of neural activity across cortical areas correlates with conscious perception. J. Neurosci. 27, 2858-2865.

Mitchell, K.J., Durstewitz, P.O., Fenton, A.A., Gingrich, J.A., Gordon, J.A., Kelsh, W., Moghaddam, B., Phillips, W.A., and Sawa, A. (2013). How can models be better utlized to enhance outcome? In: Silverstein, S.M., Moghaddam, B., Wykes, T. (Eds.), Schizophrenia: Evolution and Synthesis. MIT Press, Cambridge, MA, pp. 13-34.

Mizobe, K., Polat, U., Pettet, M. W., Kasamatsu, T. (2001). Facilitation and suppression of single striate-cell activity by spatially discrete pattern stimuli presented beyond the receptive field. Vis. Neurosci., 18, 377-391.

Moghaddam, B., \& Javitt, D. (2012). From revolution to evolution: the glutamate hypothesis of schizophrenia and its implication for treatment. Neuropsychopharmacology, 37, 4-15. doi: 10.1038/npp.2011.181

Monier, C., Chavane, F., Baudot, P., Graham, L. J., \& Fregnac, Y. (2003). Orientation and direction selectivity of synaptic inputs in visual cortical neurons: a diversity of combinations produces spike tuning. Neuron, 37, 663-680.

Muckli, L., \& Petro, L. S. (2013). Network interactions: Non-geniculate input to V1. Curr. Opin. Neurobiol, 23, 195-201.

Munneke, J.,Brentari, V., \& Peelen, M. V. (2013). The influence of scene context on object recognition is independent of attentional focus. Front. Psychol., 4, 552. doi:10.3389/fpsyg.2013. 00552.

Näätänen, R., \& Kähkönen, S. (2009). Central auditory dysfunction in schizophrenia as revealed by the mismatch negativity (MMN) and its magnetic equivalent $\mathrm{MMNm}$ : a review. Int. J. Neuropsychopharmacol., 12, 125-135. doi: 10.1017/S1461145708009322.

Neumann, H., \& Sepp, W. (1999). Recurrent V1-V2 interaction in early visual boundary processing. Biological Cybernetics, 81, 425-444.

Nithianantharajah J., Komiyama, N. H., McCechanie, A., et al. (2013). Synaptic scaffold evolution generated components of vertebrate cognitive complexity. Nat. Neurosci., 16, 16-24.

Ohzawa, I., Sclar, G., \& Freeman, R. D. (1985) Contrast gain control in the cat's visual system. $J$. Neurophysiol. 54, 651-667.

Oliva, A., \& Torralba, A. (2007). The role of context in object recognition.Trends Cogn. Sci., 11, 520-527.

Olsen, S. R., Bortone, D. S., Adesnik, H., \& Scanziani, M. (2012). Gain control by layer six in cortical circuits of vision. Nature, 483, 47-52.

Olypher, A. V., Klement, D., \& Fenton, A. A. (2006). Cognitive disorganization in hippocampus: a physiological model of the disorganization in psychosis. J Neurosci, 26, 158-168.

O'Reilly, K. C., Kao, H. Y., Lee, H., \& Fenton, A. A. (2014). Converging on a core cognitive deficit: the impact of various neurodevelopment insults on cognitive control. Front. Neurosci, 8, 153. doi: $10.3389 /$ fnins.2014.00153

Pack, C. C., \& Born, R. T. (2001). Temporal dynamics of a neural solution to the aperture problem in cortical area MT. Nature, 409, 1040-1042. 
Pack, C. C., Livingstone, M. S., Duffy, K. R., \& Born, R. T. (2003). End-stopping and the aperture problem: Two-dimensional motion signals in macaque V1. Neuron, 39, 671-680.

Palmer, L. M., Shai, A. S., Reeve, J. E., Andersen, H. L., Paulsen, O., \& Larkum, M. E. (2014). NMDA spikes enhance action potential generation during sensory input. Nat. Neurosci. 17, 383390.

Park, S., Lee, J., Folley, B., and Kim, J. (2003). Schizophrenia: Putting context in context. Behav. Brain Sci., 26, 98-99.

Patterson, J. V., Hetrick, W. P., Boutros, N. N., Jin, Y., Sandman, C., Stern, H., Potkin, S., \& Bunney, W. E., Jr. (2008). P50 sensory gating ratios in schizophrenics and controls: a review and data analysis. Psychiatry Res., 158, 226-247. doi: 10.1016/j.psychres.2007.02.009.

Perrinet, L. U., \& Masson, G. S. (2012) Motion-based prediction is sufficient to solve the aperture problem. Neural Comp. , 24, 2726-2750.

Phillips, W. A. (2012). Self-organized complexity and Coherent Infomax from the viewpoint of Jaynes's probability theory. Information, 3, 1-15, DOI 10.3390/info3010001.

Phillips, W. A. (2013). Neuronal inference must be local, selective, and coordinated. Behav. Brain Sci., 36, 222-223.

Phillips, W. A. Kay, J., \& Smyth, D. (1995). The discovery of structure by multi-stream networks of local processors with contextual guidance. Network: Comput. Neural Syst. 6, 225-246.

Phillips, W. A., \& Silverstein, S. M. (2003). Convergence of biological and psychological perspectives on cognitive coordination in schizophrenia. Behav. Brain Sci., 26, 65-138.

Phillips, W. A., \& Silverstein, S. M. (2013). The coherent organization of mental life depends on mechanisms for context-sensitive gain-control that are impaired in schizophrenia. Front.Psychol., 4:307. doi: 10.3389/fpsyg.2013.00307

Phillips, W. A., \& Singer, W. (1997). In search of common foundations for cortical computation. Behav. Brain Sci., 20, 657-722.

Phillips, W. A., von der Malsburg, C. \& Singer, W. (2010). Dynamic coordination in brain and mind. In C. von der Malsburg, W. A. Phillips, \& W. Singer (Eds.) Dynamic coordination in the brain: from neurons to mind (Strüngmann forum report: Vol. 5) (pp. 1-24). Cambridge, MA: MIT Press.

Place, E. J., \& Gilmore, G. C. (1980). Perceptual organization in schizophrenia. J. Abnorm. Psychol., 89, 409-418.

Polat, U., Mizobe, K., Pettet, M. W., Kasamatsu, T. \& Norcia, A. M. (1998). Collinear stimuli regulate visual responses depending on cell's contrast threshold. Nature, 391, 580-84.

Polat, U. \& Norcia, A. M. (1996). Neurophysiological evidence for contrast dependent long range facilitation and suppression in the human visual cortex. Vis. Res., 36, 2099-109.

Polat, U., \& Sagi D. (1993). Lateral interactions between spatial channels: Suppression and facilitation revealed by lateral masking experiments. Vis. Res. 33, 993-99.

Polat, U., \& Sagi D. (2006) Temporal asymmetry of collinear lateral interactions. Vis. Res. 46, 953960.

Pouille, F., Marin-Burgin, A., Adesnik, H., Atallah, B.V., \& Scanziani, M. (2009). Input normalization by global feedforward inhibition expands cortical dynamic range. Nat. Neurosci., $12,1577-1585$.

Pouille, F., \& Scanziani, M. (2001). Enforcement of temporal fidelity in pyramidal cells by somatic feed-forward inhibition. Science, 293, 1159-1163.

Quattrocki, E., \& Friston, K. (2014). Autism, oxytocin and interoception. Neurosci. \& Biobehav. Rev, 47, 410-430.

Qui, F. T., Sugihara, T., \& von der Heydt, R. (2007). Figure-ground mechanisms provide structure for selective attention. Nat. Neurosci., 10(11), 1492-1499.

Ramalingam, N., McManus, J. N. J., Li, W., \& Gilbert C. D. (2013). Top-down modulation of lateral interactions in visual cortex. J. Neurosci., 33, 1773-1789. 
Rasiwasia, N., \& Vasconcelos, N. (2012). Holistic context models for visual recognition. IEEETransactions on Pattern Analysis and Machine Intelligence, 34, 902-917.

Reynolds, J. H., \& Heeger, D. J. (2009). The normalization model of attention. Neuron, 61, 168-185.

Robol, V., Tibber, M. S., Anderson, E. J., Bobin, T., Carlin, P., Shergill, S. S., \& Dakin, S. C. (2013). Reduced crowding and poor contour detection in schizophrenia are consistent with weak surround inhibition. PLoS One, 8(4), e60951. doi: 10.1371/journal.pone.0060951.

Roelfsema, P.R., Lamme, V.A., \& Spekreijse, H. (1998). Object based attention in the primary visual cortex of the macaque monkey. Nature, 395, 376-381.

Rudy, B., Fishell, G., Lee, S., Hjerling-Leffler, J. (2011). Three groups of interneurons account for nearly $100 \%$ of neocortical GABAergic neurons. Dev Neurobiol. 71, 45-61.

Rust, N. C., Mante, V., Simoncelli, E. P., Movshon, J. A. (2006). How MT cells analyze the motion of visual patterns. Nat. Neurosci., 9, 1421-1431.

Rust, N. C., \& Stocker, A. A. (2010). Ambiguity and invariance: two fundamental challenges for visual processing. Curr. Opin. Neurobiol., 20, 383-388.

Ryan, T. J., Kopanitsa, M. V., Indersmitten, T., Nithianantharajah, J., Afinowi, N.,O., Pettit, C., Stanford, L. E., Sprengel, R., Saksida, L. M., Bussey, T. J., O’Dell, T. J., Grant, S. G.\& Komiyama, N. H. (2013). Evolution of GluN2A/B cytoplasmic domains diversified vertebrate synaptic plasticity and behavior. Nat Neurosci., 16, 25-32.

Salinas, E. (2004). Fast remapping of sensory stimuli onto motor actions on the basis of contextual modulation. J. Neurosci., 24, 1113-1118.

Salinas, E. (2009). Gain Modulation. In: L. R. Squire (Ed.) Encyclopedia of Neuroscience, Vol. 4 (pp. 485-490). Oxford: Academic Press.

Salinas, E., \& Sejnowski, T.J. (2001) Gain modulation in the central nervous system: Where behavior, neurophysiology, and computation meet. Neuroscientist, 7, 430-440.

Salinas, E., \& Their, P. (2000). Gain modulation: A major computational principle of the central nervous system. Neuron, 27, 15-21.

Sayim, B., Westheimer, G., \& Herzog, M. H. (2011). Quantifying target conspicuity in contextual modulation by visual search. J. Vis., 11(1):6, 1-11, doi:10.1167/11.1.6.

Schallmo, M. P., Sponheim, S. R., \& Olman, C. A. (2013). Abnormal contextual modulation of visual contour detection in patients with schizophrenia. PLoS One., 8(6), e68090. doi: 10.1371/journal.pone.0068090.

Schummers, J., Marino, J., \& Sur, M. (2002). Synaptic integration by V1 neurons depends on location within the orientation map. Neuron, 36, 969-978.

Schwartz, J. L., Grimault, N., Hupe' , J. M., Moore, B. C. J., \& Pressnitzer, D. (2012). Multistability in perception: binding sensory modalities, an overview. Philosophical Transactions of the Royal Society B-Biological Sciences, 367, 896-905.

Schwartz, O., Hsu, A., \& Dayan, P. (2007). Space and time in visual context. Nat. Rev. Neurosc., 8, $522-535$.

Schwartz, O., \& Simoncelli, E.P. (2001) Natural signal statistics and sensory gain control. Nat. Neurosci. 4, 819-825.

Seeger, M. (2013). Commentary on Martin \& Pacherie. Out of nowhere: Thought insertion, ownership and context-integration. Conscious. Cogn., 22, 261-263.

Seriès, P., Lorenceau, J., \& Frégnac, Y. (2003). The "silent" surround of V1 receptive fields: theory and experiments. $J$ Physiol. Paris, 97, 453-474.

Self, M. W., Kooijmansa, R. N., Supèr, H., Lamme, V. A., \& Roelfsema, P. R. (2012). Different glutamate receptors convey feedforward and recurrent processing in macaque V1. Proc. Natl. Acad. Sci. USA 109, 11031-11036, doi: 10.1073/pnas.1119527109.

Seth, A. K., Suzuki, K., \& Critchley, H. D. (2011). An interoceptive predictive coding model of conscious presence. Front. Psych., 2, 395. 
Seth, A. K. (2013). Interoceptive inference, emotion, and the embodied self. Trends Cogn. Sci., 17, 565-573.

Shadlen, M. N., \& Movshon, J. A. (1999). Synchrony unbound: A critical evaluation of the temporal binding hypothesis. Neuron, 24, 67-77.

Shushruth, S., Ichida, J. M., Levitt, J. B., Angelucci, A. (2009). Comparison of spatial summation properties of neurons in macaque V1 and V2. J. Neurophysiol., 102, 2069 -2083.

Silver, R. A. (2010). Neuronal arithmetic. Nat. Rev. Neurosci., 11, 474-489.

Siegel, M., Körding, K. P. \& König, P. (2000). Integrating top-down and bottom-up sensory processing by somato-dendritic interactions. J. Comput. Neurosci., 8, 161-173.

Silverstein, S. M. (2010). Failures of dynamic coordination in disease states, and their implications for normal brain function. In C. von der Malsburg, W. A. Phillips, \& W. Singer (Eds.) Dynamic coordination in the brain: from neurons to mind (Strüngmann forum report: Vol. 5) (pp. 245265). Cambridge, MA: MIT Press.

Silverstein, S. M., Berten, S., Essex, B., Kovács, I., Susmaras, T., \& Little, D. M. (2009). An fMRI examination of visual integration in schizophrenia. J. Integr. Neurosci., 8, 175-202.

Silverstein, S. M. \& Keane, B. P. (2011). Perceptual organization impairment in schizophrenia and associated brain mechanisms: review of research from 2005 to 2010. Schizophr. Bull., 37, 690699. doi: 10.1093/schbul/sbr052.

Silverstein, S. M., Keane, B. P., Wang, Y., Mikkilineni, D., Paterno, D., Papathomas, T. V., \& Feigenson, K. (2013). Effects of short-term inpatient treatment on sensitivity to a size contrast illusion in first-episode psychosis and multiple-episode schizophrenia. Front Psychol., 4:466. doi: 10.3389/fpsyg.2013.00466.

Silverstein, S. M., \& Uhlhaas, P. J. (2004). Gestalt psychology: The forgotten paradigm in abnormal psychology. Am. J. Psych., 117, 259-277.

Simoncelli, E. P., \& Schwartz, O. (1999). Modeling Surround Suppression in V1 Neurons with a Statistically-Derived Normalization Model. In M. S. Kearns, S. A. Solla, \& D. A. Cohn (Eds.) Advances in Neural Information Processing Systems (pp. 153-159). Cambridge, MA.: MIT Press, 153-159.

Singer, W. (1999). Neural synchrony: A versatile code for the definition of relations. Neuron, 24, 49-65.

Smyth, D., Phillips, W. A., \& Kay, J. (1996). Measures for investigating the contextual modulation of information transmission. Network: Computation in Neural Systems, 7, 307-316.

Sohal, V. S., Zhang F., Yizhar, O., Deisseroth, K. (2009). Parvalbumin neurons and gamma rhythms enhance cortical circuit performance. Nature, 459, 698-702.

Solomon, J. A., \& Morgan, M. J. (2000). Facilitation from collinear flanks is cancelled by noncollinear flanks. Vis. Res., 40, 279-286.

Spratling, M. W. (2008). Predictive-coding as a model of biased competition in visual attention. Vis. Res., 48, 1391-1408.

Spratling, M. W. (2010). Predictive coding as a model of response properties in cortical area V1. J. Neurosci, 30, 3531-43.

Spratling, M. W. (2013). Distinguishing theory from implementation in predictive coding accounts of brain function. Behav. Brain Sci., 36, 231-232.

Spratling, M. W. (2014). A single functional model of drivers and modulators in cortex. J. Comput. Neurosci., 36, 97-118.

Spratling, M. W., De Meyer, K., \& Kompass, R. (2009). Unsupervised learning of overlapping image components using divisive input modulation. Comput. Intell. Neurosci.,1-19. doi:10.1155/2009/381457

Spratling, M.W. and Johnson, M. H. (2004). A feedback model of visual attention. J. Cogn. Neurosci., 16, 219-37. 
Spratling, M. W. and Johnson, M. H. (2006). A feedback model of perceptual learning and categorisation. Vis. Cogn., 13, 129-65.

Spruston, N. (2008). Pyramidal neurons: dendritic structure and synaptic integration. Nat. Rev. Neurosci. 9, 206-221. doi:10.1038/nrn2286

Stettler, D. D., Das, A., Bennett, J., Gilbert, C. D. (2002). Lateral connectivity and contextual interactions in macaque primary visual cortex. Neuron 36, $739-750$.

Sugita, Y. (1999). Grouping of image fragments in primary visual cortex. Nature, 401, 269-272.

Thiele, A, Pooresmaeili, A., Delicato, L. S., Herrero, J. L., and Roelfsema P. R. (2009). Additive effects of attention and stimulus contrast in primary visual cortex. Cerebral Cortex, 19, 29702981. doi:10.1093/cercor/bhp070

Tibber, M. S., Anderson, E. J., Bobin, T., Antonova, E., Seabright, A., Wright, B., Carlin, P., Shergill, S. S., \& Dakin, S. C. (2013). Visual surround suppression in schizophrenia. Front. Psychol., 4, 88. doi: 10.3389/fpsyg.2013.00088.

Tiesinga, P. J., Fellous, J-M., and Sejnowski, T. J. (2008). Regulation of spike timing in visual cortical circuits. Nat. Rev. Neurosci. 9, 97-109.

Torralba, A. (2009) How many pixels make an image? Vis. Neurosci., 26(1):123-13.

Tregellas, J. R., Smucny, J., Eichman, L., \& Rojas, D. C. (2012). The effect of distracting noise on the neuronal mechanisms of attention in schizophrenia. Schizophr Res, 142, 230-236

Treue, S., \& Martinez Trujillo, J.C. (1999). Feature-based attention influences motion processing gain in macaque visual cortex. Nature, 399, 575-579.

Uhlhaas, P. J., Phillips, W. A., Mitchell, G., \& Silverstein, S. M. (2006) Perceptual grouping in disorganized schizophrenia. Psychiatry Research 145, 105-117.

Uhlhaas, P. J., Phillips, W. A., \& Silverstein, S. M. (2005). The course and clinical correlates of dysfunctions in visual perceptual organization in schizophrenia during the remission of psychotic symptoms. Schizophr. Res., 75, 183-192.

Uhlhaas, P. J., Pipa, G., Lima, B., Melloni, L., Neuenschwander, S., Nikolic, D., Singer, W. (2009). Neural synchrony in cortical networks: history, concept and current status. Front. Integr. Neurosci., 3, 1-19.10.3389/neuro.07.017.2009

Uhlhaas, P. J., \& Silverstein, S. M. (2005). Perceptual organization in schizophrenia spectrum disorders: empirical research and theoretical implications. Psychol. Bull., 131, 618-632.

Uhlhaas, P. J., \& Singer, W. (2010). Abnormal neural oscillations and synchrony in schizophrenia. Nat. Rev. Neurosci., 11, 100-113. doi: 10.1038/nrn2774.

Uhlhaas, P. J., \& Singer, W. (2013). High-frequency oscillations and the neurobiology of schizophrenia. Dialogues Clin. Neurosci., 15, 301-313.

Urban, A., Kremlácek, J., Masopust, J., \& Libiger, J. (2008). Visual mismatch negativity among patients with schizophrenia. Schizophr. Res., 102, 320-328. doi: 10.1016/j.schres.2008.03.014.

Vinck, M., Womelsdorf, T., Fries, P. (2013) Gamma-band synchronization and information transmission. In R. Q. Quiroga \& S. Panzeri (Eds.) Principles of Neural Coding (pp. 449-469). London: CRC Press.

von der Malsburg, C., Phillips, W. A., \& Singer, W. (Eds.) (2010). Dynamic coordination in the brain: from neurons to mind. Strüngmann forum report: Vol. 5. Cambridge, MA: MIT Press.

Walther, S., Ramseyer, F., Horn, H., Strik, W., \& Tschacher W. (2014). Less structured movement patterns predict severity of positive syndrome, excitement, and disorganization. Schizophr Bull., 40, 585-91. doi: 10.1093/schbul/sbt038

Ward, P. B., Catts, S. V., Fox, A. M., Michie, P. T., \& McConaghy, N. (1991). Auditory selective attention and event-related potentials in schizophrenia. Psychiatry, 158, 534-539.

Waters, J., Larkum, M., Sakmann, B., and Helmchen, F. (2003). Supralinear $\mathrm{Ca}^{2}$ influx into dendritic tufts of layer 2/3 neocortical pyramidal neurons In Vitro and In Vivo. J. Neurosci., 23, 85588567. 
Watt, R. J., \& Phillips, W. A. (2000). The function of dynamic grouping in vision. Trends Cogn. Sci., 4, 447-454.

Wesierska, M., Dockery, C., and Fenton, A. A. (2005) Beyond memory, navigation, and inhibition: behavioral evidence for hippocampus-dependent cognitive coordination in the rat. J. Neurosci., $25,2413-2419$.

Williford, T., \& Maunsell, J.H. (2006). Effects of spatial attention on contrast response functions in macaque area V4. J. Neurophysiol., 96, 40-54.

Wilson, N. R., Runyan, C. A., Wang, F.L., \& Sur,M. (2012). Division and subtraction by distinct cortical inhibitory networks in vivo. Nature, 488, 343-348.

Wishner, J., \& Wahl, O. (1974). Dichotic listening in schizophrenia. J Consult Clin Psychol, 42, 538-546.

Yoon, J. H., Maddock, R. J., Rokem, A., Silver, M. A., Minzenberg, M. J., Ragland, J. D., \& Carter, C. S. (2010). GABA concentration is reduced in visual cortex in schizophrenia and correlates with orientation-specific surround suppression. J. Neurosci., 30, 3777-3781.

Yoon, J. H, Rokem, A. S., Silver, M. A., Minzenberg, M. J., Ursu, S., Ragland, J. D., \& Carter CS. (2009). Diminished orientation-specific surround suppression of visual processing in schizophrenia. Schizophr. Bull., 35, 1078-1084. doi: 10.1093/schbul/sbp064.

Yoon, J. H., Sheremata, S. L., Rokem, A., \& Silver, M. A. (2013). Windows to the soul: vision science as a tool for studying biological mechanisms of information processing deficits in schizophrenia. Front.Psychol., 4, 681. doi:10.3389/fpsyg. 2013.0068

Zhou, Z, Bernard, MR, Bonds, AB (2008). Deconstruction of spatial integrity in visual stimulus detected by modulation of synchronized activity in cat visual cortex. J. Neurosci., 28, 37593768 . 
